\title{
Understanding storm-time ring current development through data-model comparisons of a moderate storm
}

\author{
Jichun Zhang, ${ }^{1,2}$ Michael W. Liemohn, ${ }^{1}$ Darren L. De Zeeuw, ${ }^{1}$ Joseph E. Borovsky, ${ }^{3}$ \\ Aaron J. Ridley, ${ }^{1}$ Gabor Toth, ${ }^{1}$ Stanislav Sazykin, ${ }^{4}$ Michelle F. Thomsen, ${ }^{3}$ \\ Janet U. Kozyra, ${ }^{1}$ Tamas I. Gombosi, ${ }^{1}$ and Richard A. Wolf ${ }^{4}$ \\ Received 11 May 2006; revised 22 November 2006; accepted 4 December 2006; published 18 April 2007.
}

[1] With three components, global magnetosphere (GM), inner magnetosphere (IM), and ionospheric electrodynamics (IE), in the Space Weather Modeling Framework (SWMF), the moderate storm on 19 May 2002 is globally simulated over a 24-hour period that includes the sudden storm commencement (SSC), initial phase, and main phase of the storm. Simulation results are validated by comparison with in situ observations from Geotail, GOES 8, GOES 10, Polar, LANL MPA, and the Sym- H and Dst indices. It is shown that the SWMF is reaching a sophistication level for allowing quantitative comparison with the observations. Major storm characteristics at the SSC, in the initial phase, and in the main phase are successfully reproduced. The simulated plasma parameters exhibit obvious dawn-dusk asymmetries or symmetries in the ring current region: higher density near the dawn and higher temperature in the afternoon and premidnight sectors; the pressure is highest on the nightside and exhibits a near dawn-dusk symmetry. In addition, it is found in this global modeling that the upstream solar wind/ IMF conditions control the storm activity and an important plasma source of the ring current is in the solar wind. However, the ionospheric outflow can also affect the ring current development, especially in the main phase. Activity in the high-latitude ionosphere is also produced reasonably well. However, the modeled cross polar cap potential drop (CPCP) in the Southern Hemisphere is almost always significantly larger than that in the Northern Hemisphere during the May storm.

Citation: Zhang, J., et al. (2007), Understanding storm-time ring current development through data-model comparisons of a moderate storm, J. Geophys. Res., 112, A04208, doi:10.1029/2006JA011846.

\section{Introduction}

[2] A geomagnetic storm is primarily caused by a geoeffective solar wind structure containing a large, prolonged, southwardly directed interplanetary magnetic field (IMF) [e.g., Gonzalez et al., 1994, and references therein; Zhang et al., 2004]. The dayside magnetic reconnection [Dungey, 1961] between the southward IMF and the geomagnetic field enhances solar wind mass transport and energy transfer into the magnetosphere [Gonzalez et al., 1994; Borovsky et al., 1997, 1998b]. Meanwhile, ionospheric cold plasma can also make a contribution to the hot plasma of the magnetosphere through outflow from the auroral zone at high latitudes in response to changes in the solar wind [Shelley et al., 1972; Lennartsson, 1997; Fuselier et al., 2003, and

\footnotetext{
${ }^{1}$ Center for Space Environment Modeling, University of Michigan, Ann Arbor, Michigan, USA.

${ }^{2}$ Now at Department of Physics and Astronomy, Rice University, Houston, Texas, USA.

${ }^{3}$ Los Alamos National Laboratory, Los Alamos, New Mexico, USA.

${ }^{4}$ Department of Physics and Astronomy, Rice University, Houston, Texas, USA.

Copyright 2007 by the American Geophysical Union. 0148-0227/07/2006JA011846
}

references therein]. However, the relative importance of solar wind and ionospheric plasma sources is still unclear. Under the enhanced convection electric fields and geomagnetic fields, a mixture of the fresh ionospheric and solar wind plasmas, together with the magnetospheric plasmas, is energized and undergoes large-scale drift in the global magnetosphere. Some of the particles can be transported inward enough to form the storm-time ring current. The ring current, typically at radial distances of about 2-7 Earth radii $\left(R_{E}\right)$, consists of $10-200 \mathrm{keV}$ ions (mainly $\mathrm{H}^{+}$, and $\mathrm{O}^{+}$) and electrons that undergo an azimuthal drift around the Earth [e.g., Frank, 1967; Gonzalez et al., 1994; Daglis et al., 1999, and references therein].

[3] To explore the direct source for the storm-time ring current, Zhang et al. [2006] analyzed hot-ion (in the energy range $0.1-45 \mathrm{keV}$ ) measurements at geosynchronous orbit from the Los Alamos Magnetospheric Plasma Analyzer (MPA) with the superposed epoch technique. Figure 1 shows superposed average values of hot-ion number density, temperature, and pressure during 63 moderate storms at solar maximum as a function of local time (LT) and universal time (UT). For the purpose of comparison, the means (solid line) and medians (dotted line) of $D s t^{*}$ (pressure-corrected Dst) are also plotted; the first and third 


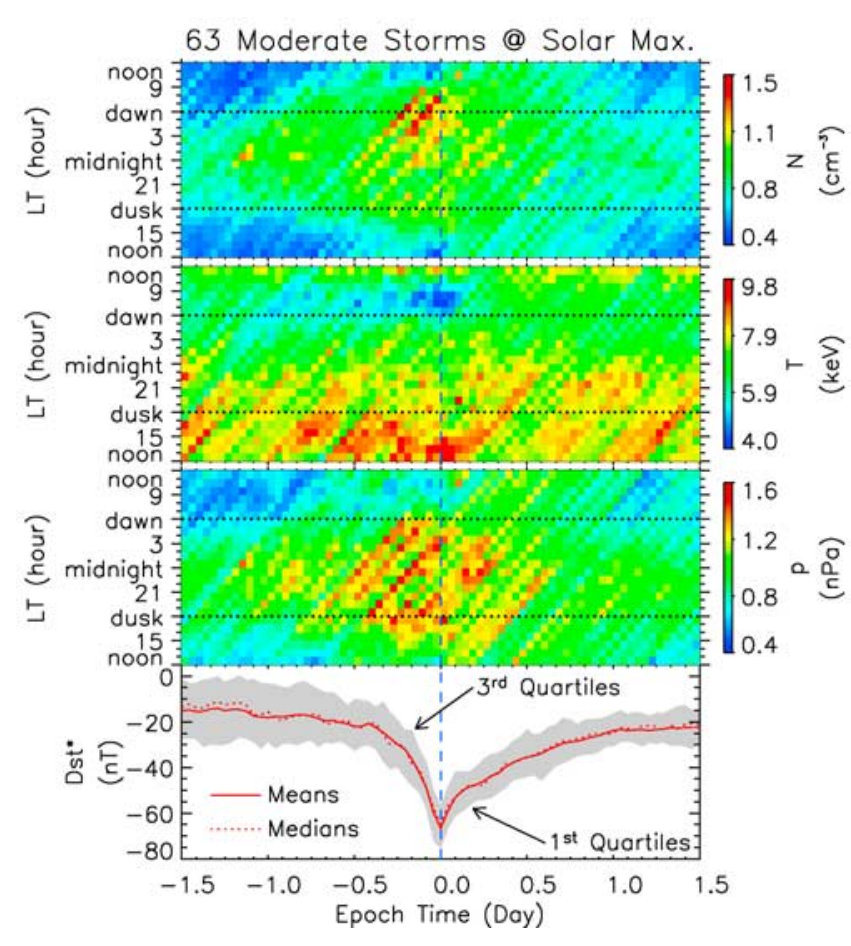

Figure 1. Superposed epoch averages for 63 moderate storm events at solar maximum. From top to bottom are three LT-UT maps of color-coded average MPA measurements at geosynchronous orbit and means (solid) and medians (dotted) of $D s t^{*}$ variation with first and third quartiles shown by the lower and upper boundaries of the shading. The three geosynchronous-orbit observations are hot-ion $(0.1-45 \mathrm{keV})$ number density $\left(N\right.$, in $\left.\mathrm{cm}^{-3}\right)$, temperature $(T$, in $\mathrm{keV})$, and pressure $(p$, in $\mathrm{nPa})$. Zero on the epoch time axis, denoted by the vertical dashed line, corresponds to minimum $D s t^{*}$. The nightside is between the two horizontal dotted lines in the panels of the MPA parameters. This figure is adapted from Figure 5 of Zhang et al. [2006].

quartiles are indicated by the lower and upper boundaries of the shading, respectively. Zero epoch time is selected at the minimum $D s t^{*}$, which is denoted by the vertical dashed line. Between the two horizontal dotted lines in the panels of the MPA parameters is the nightside. Figure 1 shows that around the minimum $D s t^{*}$ the density is more enhanced near dawn and temperature is higher in the afternoon and premidnight sectors; however, the pressure peaks on the nightside and exhibits approximate dawn-dusk symmetry during the storm main phase. One purpose of this study is to investigate how the LT dependence of the MPA parameters at geosynchronous orbit affects the development of the storm-time ring current.

[4] The other purpose of the present study is to test the performance of a newly developed Space Weather Modeling Framework, in short, SWMF [Tóth et al., 2004, 2005]. In this study, an actual space weather event, the moderate storm on 19 May 2002, is simulated globally with the SWMF. Extensive data model comparisons are made to quantify the accuracy of the simulation results. Note that during the storm event almost continuous in situ observa- tions are available in the upstream solar wind, in the global magnetosphere, and at geosynchronous orbit.

[5] The paper is organized in the following way: section 2 briefly introduces the modeled moderate storm, reviews the SWMF, and describes how the boundary and initial conditions for the run were selected; section 3 presents the simulation results, including modeling verification and simulated activity in the inner magnetosphere and polar regions; the conclusions from the storm simulation are stated in section 4 with relevant discussion and are then summarized in section 5 .

\section{Simulation}

\subsection{The 19 May 2002 Storm Event and Solar Wind Input}

[6] The minimum Dst (Dst $\left.t_{\min }\right)$ of the selected moderate storm is $-58 \mathrm{nT}$ at 0600 UT on DOY 139 (19 May) of 2002. Figure 2 shows the upstream solar wind conditions observed by the ACE spacecraft and $S y m-H$ and $D s t$ in the bottom panel on the second half of 18 May and the first half of 19 May 2002. With the identification criteria for a magnetic cloud listed in the work of Burlaga [1991] and Zhang et al. [2004], the solar wind driver of this storm can be defined as a "bipolar $B_{z}$ " magnetic cloud (MC), which is denoted by the second and third vertical solid lines in Figure 2. Because the magnetic field in the cloud rotates smoothly from southward to northward (see $B_{z}$ panel in Figure 2), it can also be called an SN magnetic cloud. Ahead of the cloud event are an obvious shock, indicated by the first vertical solid line, and a sheath with highly disturbed solar wind parameters. The IMF $B_{z}$ is southward or geoeffective both in the sheath and in the leading field of the cloud. The horizontal dashed line in the $B_{z}$ panel indicates the thresholds ( $B_{z} \leq-5 \mathrm{nT}$ for more than 2 hours) for moderate storms $\left(-100 \mathrm{nT}<D s t_{\min } \leq-50 \mathrm{nT}\right)$ at the $80 \%$ occurrence level [Gonzalez and Tsurutani, 1987; Gonzalez et al., 1994]. Responding to these two geoeffective regions, Sym- $H$ and Dst drop apparently both in the early and late main phase.

[7] The storm sudden commencement (SSC) of the storm is indicated by sharp rise in $S y m-H$ from $2 \mathrm{nT}$ to $57 \mathrm{nT}$ in $6 \mathrm{~min}$, even though Dst peaks only at $26 \mathrm{nT}$. This SSC is driven by the solar wind shock wave mentioned above: the solar wind speed increases from $\sim 350$ to almost $500 \mathrm{~km} / \mathrm{s}$; density is tripled to $30 \mathrm{~cm}^{-3}$ with a peak at $51 \mathrm{~cm}^{-3}$; and the corresponding peak dynamic pressure (not shown) is as high as $20 \mathrm{nPa}$.

[8] Note that the solar wind and IMF data in Figure 2 were propagated from the ACE location, at near the Lagrange-1 point, to $(32,0,0) R_{E}$ in GSM coordinates with a sophisticated propagation method, the minimum variance analysis (MVA) technique [Weimer et al., 2003; Weimer, 2004]. The eight solar wind parameters plotted in Figure $2, B_{x}, B_{y}, B_{z}, V_{x}, V_{y}, V_{z}, n$, and $T$, together with an adiabatic index $\gamma=5 / 3$, are the solar wind input to the SWMF. However, to force agreement between the time of the shock arrival and the resulting SSC observed in Sym- $H$, the propagation time to $32 R_{E}$ derived using the MVA method is decreased by $4 \mathrm{~min}$. The plane of GSM-X = $32 R_{E}$ is the outer boundary at the sunward side of the simulation domain (see below). The simulation was run 


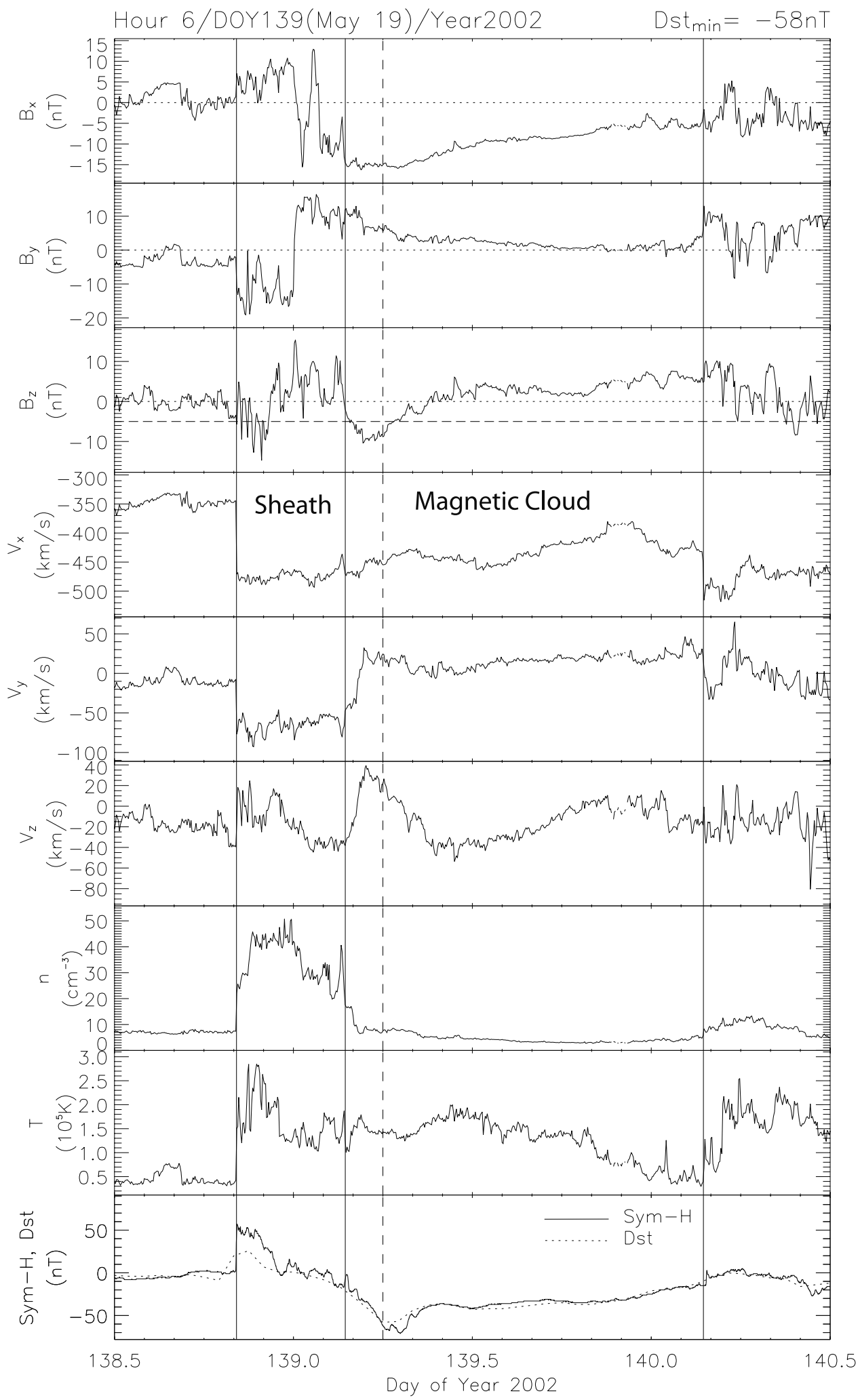

Figure 2. Propagated solar wind and IMF measurements by the ACE spacecraft, $S y m-H$ and Dst for the simulated moderate storm. From top to bottom are plotted IMF components $\left(B_{x}, B_{y}\right.$, and $\left.B_{z}\right)$, plasma velocity vector $\left(V_{x}, V_{y}\right.$, and $\left.V_{z}\right)$, proton number density $(n)$, proton temperature $(T)$, and geomagnetic indices Dst denoted by the dotted line and Sym-H by the solid line. The time of the minimum Dst index is denoted by the vertical dashed line in all panels and also shown in hour, DOY, and year order at the top with the $D s t_{\min }$ value. The horizontal dashed line in the $B_{z}$ panel denotes the thresholds for moderate storms at the 80\% occurrence level [Gonzalez and Tsurutani, 1987; Gonzalez et al., 1994]. 
with the SWMF for 24 hours, from 1200 UT on 18 May to 1200 UT on 19 May. In other words, about 8 hours of quiet time before the storm, the initial phase, the main phase, and the early recovery phase of the storm are modeled.

\subsection{Model Description: GM, IM, IE, and Their Couplings}

[9] The SWMF is a collection of nine modules, modeling nine physics domains from the surface of the Sun to the upper atmosphere of the Earth in this case, although SWMF modules have been developed for the other planets as well. The nine physics domains for the Sun-Earth interaction include (1) solar corona (SC), (2) eruptive event generator (EE), (3) inner heliosphere (IH), (4) solar energetic particles (SP), (5) global magnetosphere (GM), (6) inner magnetosphere (IM), (7) radiation belt (RB), (8) ionosphere electrodynamics (IE), and (9) upper atmosphere (UA) [Tóth et al., 2004, 2005]. For the purpose of this study, only GM, IM, and IE are chosen to do the storm simulation at the usually selected planet, the Earth.

[10] The GM component is a three-dimensional (3-D) global MHD model for which the Block-Adaptive-Tree Solar-Wind Roe-Type Upwind Scheme (BATS-R-US) code is used. The algorithms and numerical schemes of the GM/ BATS-R-US model are described in detail by Powell et al. [1999] and De Zeeuw et al. [2000]. In short, the BATS-RUS solves the ideal and nonrelativistic magnetohydrodynamic (MHD) equations to compute the magnetic field, currents, plasma density, pressure, and velocity throughout the magnetosphere. While other techniques are being developed [e.g., Nishikawa, 2001; Delamere et al., 2001], global MHD codes are widely used to theoretically represent the outer magnetosphere [e.g., Ogino and Walker, 1984; Lyon et al., 1986; Winglee, 1994; Raeder et al., 1995; Tanaka, 1995; Janhunen, 1996; White et al., 1998; Powell et al., 1999].

[11] However, the physics of the Earth's inner magnetosphere is complicated, since it contains overlapping particle distributions with a large range of energies. These different coexisting particle populations cannot be treated as a single fluid as those plasmas in the outer magnetosphere because gradient and curvature drifts are energy and velocity dependent. To achieve significant numerical accuracy and improvements in performance in the simulation of the inner magnetosphere, the Rice Convection Model (RCM) is taken as the IM component. The RCM calculates the dynamic behavior of the inner-magnetospheric particles and the electric currents and associated electric fields on a 2-dimensional (2-D) spherical grid in the ionosphere; the ionospheric results are mapped along field lines into the magnetosphere. It solves the equations of magnetospheric plasma motion for multiple isotropic "fluids," typically on the order of 100 , in a region of the magnetic equatorial plane that extends from just inside the dayside magnetopause to the nightside inner plasma sheet $\left(\sim 10 R_{E}\right)$ and to $\sim 7 R_{E}$ at dawn and dusk. Each fluid is really a volume of phase space, characterized by energy invariant, flux tube content, and charge. The distribution function is assumed constant along each magnetic field line. In the RCM, internal losses are usually assumed to be charge exchange for ions and precipitation into a loss cone for electrons [Wolf et al., 1991; Toffoletto et al., 2003]. In the present study, however, no losses are included except for the large-scale plasma convection which brings particles to the dayside magnetopause. More detailed RCM descriptions of the model equations and the numerical techniques used to solve them can be found in the work of Sazykin [2000, and references therein] and in the recent review of Toffoletto et al. [2003].

[12] It is well known that the magnetosphere and ionosphere are closely coupled with each other. The magnetospheric currents flow into and out of the conductive ionosphere along magnetic field lines or via region 1 (at higher latitudes) and region 2 (equatorward of region 1) field-aligned currents [Iijima and Potemra, 1976]. In the current version of the SWMF, the IE component the Ridley Ionosphere Model (RIM) [see Ridley et al., 2004]. The RIM is a 2-D spherical ionospheric electric potential solver using a dynamically defined conductance pattern, which captures both the conductivity and electric field in the ionosphere. The conductivity is driven primarily by sunlight on the dayside and the aurora in the high latitude region. Ridley et al. [2001] and Liemohn et al. [2005] found that comparisons of the IE/RIM results against previous studies and ionospheric data for several events showed reasonable agreement.

[13] Coupling is achieved via near-continuous two-way flow of information among the GM, IE, and IM components. De Zeeuw et al. [2004] presented initial simulation results with the coupled code under idealized conditions, such as uniform ionospheric conductance, steady solar wind, north-to-south sharply flipped IMF, and the Earth's dipole perpendicular to the solar wind flow and parallel to the rotation axis. As illustrated in Figure 3, the GM, IE, and IM are linked together as follows [De Zeeuw et al., 2004; Tóth et al., 2004, 2005]:

[14] 1. GM and IM are self-consistently coupled. GM calculates the time-dependent magnetic field inside the IM's modeling region as well as the outer magnetosphere. GM indicates whether IM grid points are on open or closed field lines; open field lines are ignored. GM provides IM with the $\mathrm{X}$ and $\mathrm{Y}$ coordinates where the field line intersects the magnetic equatorial plane (i.e., the X-Y plane in the GSM coordinates), the magnetic field magnitude, the magnetic flux tube volumes, and the average density and pressure in the flux tubes. The first three terms are denoted by X Equatorial, Y Equatorial, and B Equatorial in Figure 3, respectively. Meanwhile, IM derives its plasma distribution outer boundary from GM-computed field-line-averaged pressure and density. IM supplies the time-evolving plasma density and pressure on its 2-D spherical grid to nudge the GM values.

[15] 2. GM and IE are coupled in two ways. The fieldaligned currents, which are calculated in GM at $3.5 R_{E}$, are mapped along the dipole field lines of the Earth to the ionosphere on IE's 2-D spherical grid. The electric potential, which is computed on the 2-D spherical grid of IE, is mapped back to the inner boundary of GM (at $2.5 R_{E}$ in this study) and used to calculate the electric field and velocity at the inner boundary.

[16] 3. There is only a one way coupling between IM and IE. IE provides the electric potential for the 2-D spherical grid of IM.

[17] The couplings of the three components can also be described in terms of the electromagnetic coupling and the material coupling. For the electromagnetic coupling, GM gives the magnetic field information to IM, and the field- 


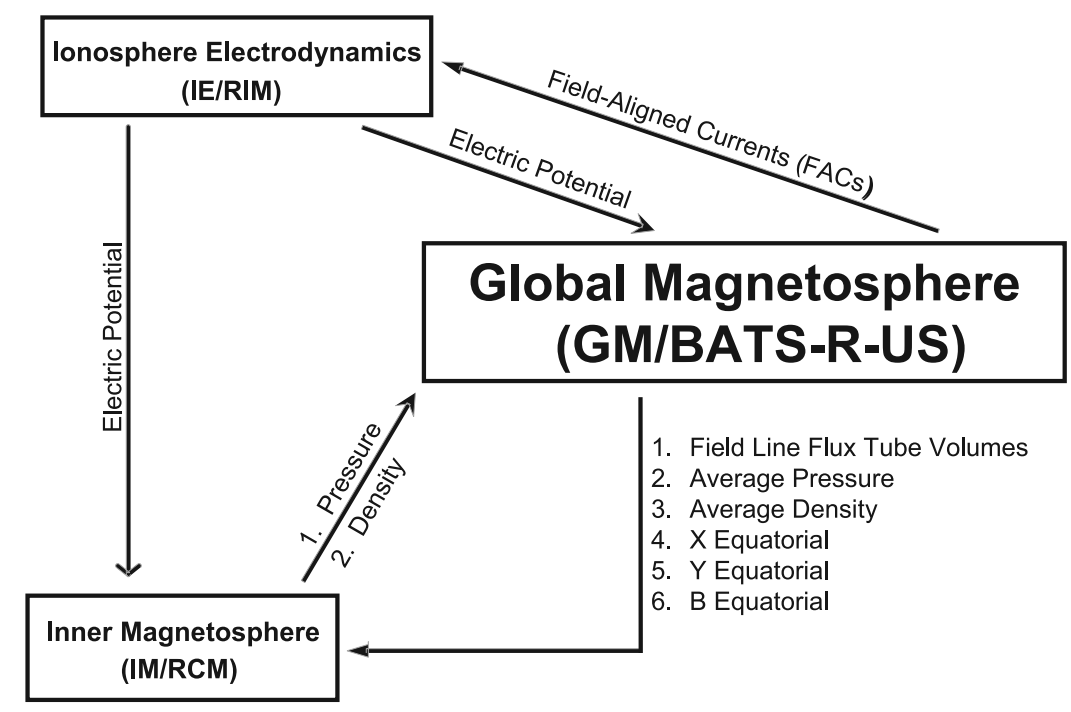

Figure 3. Couplings of GM/BATS-R-US (Block Adaptive Tree Solar-Wind Roe-Type Upwind Scheme), IM/RCM (Rice Convection Model), and IE/RIM (Ridley Ionosphere Model). See text for details.

aligned currents to IE, and IE gives the electric potential to both GM and IM. For the material coupling, GM exchanges the plasma parameters (density and pressure) with IM.

\subsection{Boundary and Initial Conditions: Run Setup}

[18] In this study, the inner boundary is placed at the radial distance of $2.5 R_{E}$ from the center of the Earth to avoid the extremely strong magnetic field inside this radius. At the inner boundary, the temperature is held constant, $25,000 \mathrm{~K}$, or $1.25 \mathrm{eV}$, and the mass density is also fixed to a value from 5 to $28 \mathrm{amu} / \mathrm{cm}^{3}$, depending on the run. In the storm simulation, the Earth's dipole strength is characterized by the magnetic field magnitude at the equator, which is about $31,100 \mathrm{nT}$ at $1 R_{E}$. The tilt angle of the dipole is

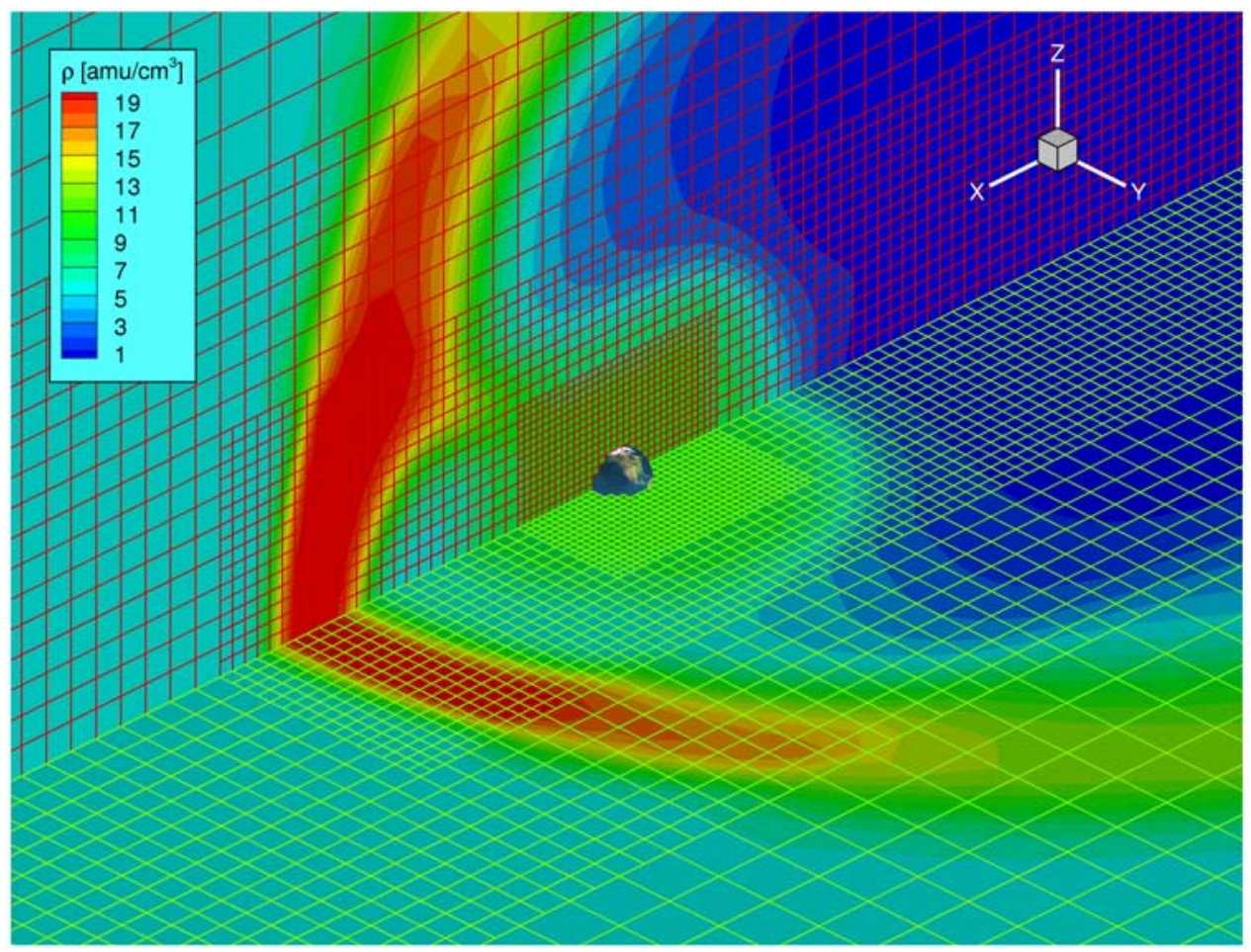

Figure 4. Close-up view of the initial steady state solution obtained by the Global Magnetosphere (GM/ BATS-R-US). Only the GSM-XZ and GSM-XY planes, color-coded by plasma mass density, are shown. The inner boundary density is held constant at $10 \mathrm{amu} / \mathrm{cm}^{3}$ in this run. The closer to the Earth, the higher the computational cell resolution is; the smallest cell is $1 / 4 R_{E}$. 
always set as in reality. The observed upstream solar wind flows in the plane of GSM-X $=32 R_{E}$, and a zero gradient condition is applied at the other walls of the outer boundary.

[19] The computational domain in the global simulation ranges from -224 to $32 R_{E}$ in the $\mathrm{X}$ direction and -64 to $64 R_{E}$ in the $\mathrm{Y}$ and $\mathrm{Z}$ directions in the GSM coordinate system. The GM/BATS-R-US code uses a block-based adaptive grid [Powell et al., 1999; De Zeeuw et al., 2000]. The grid of the computational domain is composed of 576 blocks. Each block contains $8 \times 8 \times 8$ rectangular Cartesian cells, so the computational cells in the grid total to 294,912. The cell sizes, however, vary between blocks. The coarsest blocks have cubic cells with a size of $4 R_{E}$, while the cell size is $1 / 4 R_{E}$ in the finest blocks. As shown in Figure 4, the finer cells are concentrated closer to the Earth and at the expected locations of the bow shock and the magnetotail. Note that in this simulation the cell spatial resolution is set low $\left(2.0 R_{E}\right)$ in the far magnetotail to avoid too much computing time.

[20] The storm simulation was carried out in three stages. First, the GM component runs 2000 iterations to converge to a steady state solution. Figure 4 shows a close-up view of the GM steady state in the XZ and XY planes of the GSM coordinate system. Major characteristics of the magnetosphere, such as the bow shock, magnetopause, and magnetotail, are reproduced. Second, the GM, IM, and IE components start from the GM solution and run 4000 more steps to obtain an initial steady state for all of them. In the first two stages, the code is driven by the propagated ACE observations at the modeling start time, or at noon on 18 May 2002 as indicated above. The ACE measurements are applied to the inflow boundary during the process of the two steady-state simulations, and the initial magnetic field contains only the dipole field. Simulation results presented below are all from this stage.

[21] In the IM/RCM component used in this study, protons are the only ions and losses like charge exchange are not included. It is important here to note that the flow-out loss of partial ring current ions on open drift paths, which is important during the main and early recovery phase of the storm when the IMF is still southward, is well represented in SWMF. However, when the recovery phase begins, chargeexchange loss becomes increasingly important and dominates when the IMF turns northward. This is the reason that the modeling end time is selected just after the early recovery phase of the storm. In the IE component, on the basis of the observed data, the $10.7 \mathrm{~cm}$ solar radio flux (F10.7) is set at $167.0 \times 10^{4}$ Janskys (Jy). The nightside Pedersen conductance is 0.25 mho, which dominates on the nightside except for the aurora. The average Pedersen conductance in the Polar Cap (Lat: $\sim 65^{\circ}-90^{\circ}$ ) is set at 1.0 mho.

\section{Results}

\subsection{Validations}

\subsubsection{Geotail, GOES 8, GOES 10, and Polar}

[22] During the storm event, the Geotail, GOES 8, GOES 10, and Polar spacecrafts made continuous observations in the magnetosphere. Respectively, Figures 5-7 show their measurements, marked by the dashed lines, and the comparisons with the simulation results, indicated by the solid lines. All data are in GSM coordinates. In each figure, at the
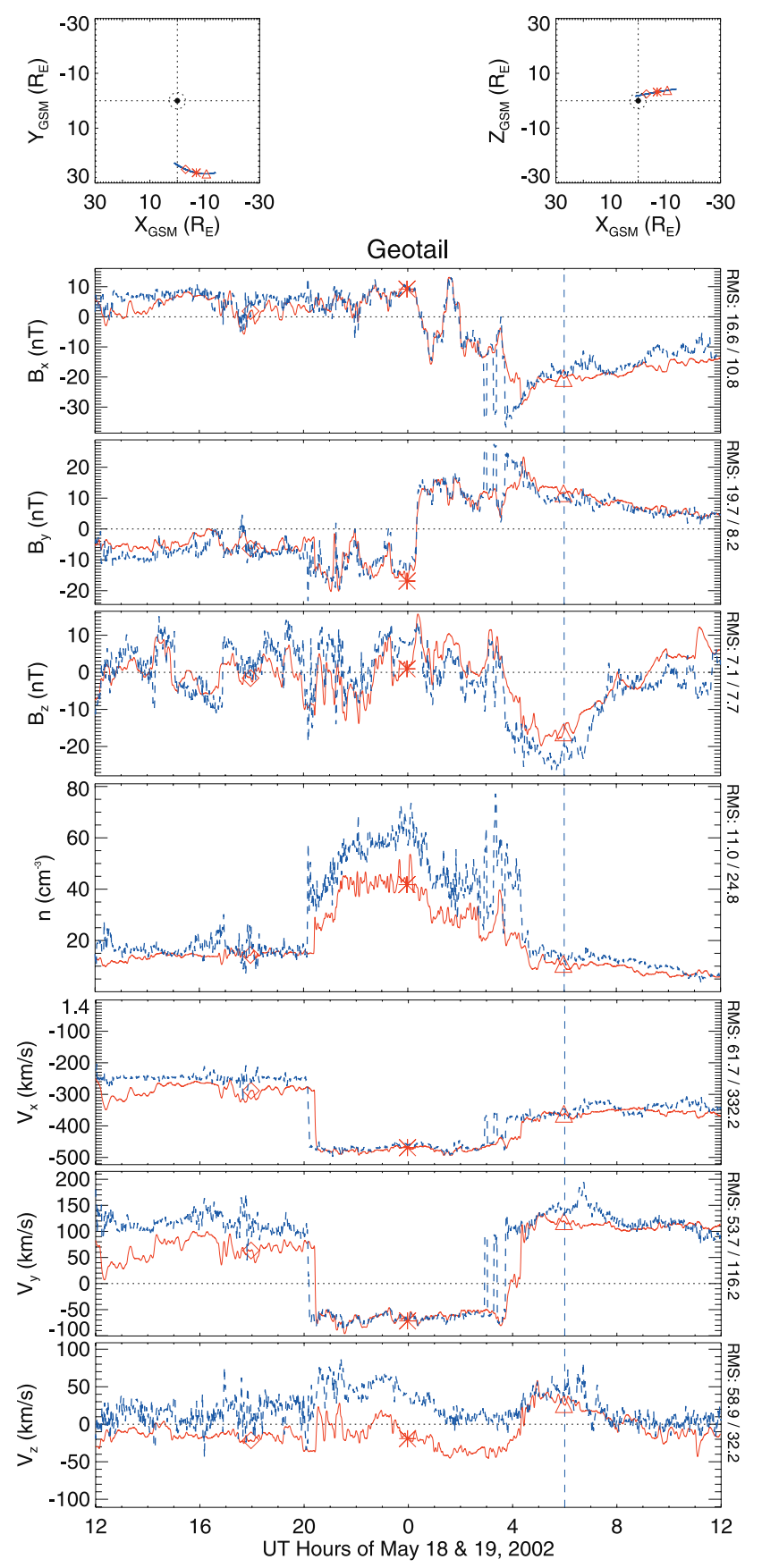

Figure 5. Geotail magnetic field and plasma data (dashed lines) and comparisons with the simulation results (solid lines) on the second half of 18 May and the first half of 19 May 2002. From top to bottom are the trajectories of Geotail in the GSM-XY and GSM-XZ planes and plotted magnetic field components $\left(B_{x}, B_{y}\right.$, and $\left.B_{z}\right)$, plasma number density $(n)$, and plasma velocity components $\left(V_{x}, V_{y}\right.$, and $\left.V_{z}\right)$ in GSM coordinates. The vertical dashed line in each panel indicates min Dst. The root-mean-square (RMS) difference between measurements and simulated data and the RMS of measurements are indicated on the right in each panel before and after the slash sign, respectively. Three symbols, a square, an asterisk, and a triangle, are illustrated in the two trajectory panels and each parameter panel to show the locations and universal times at which three sample data points are observed. 

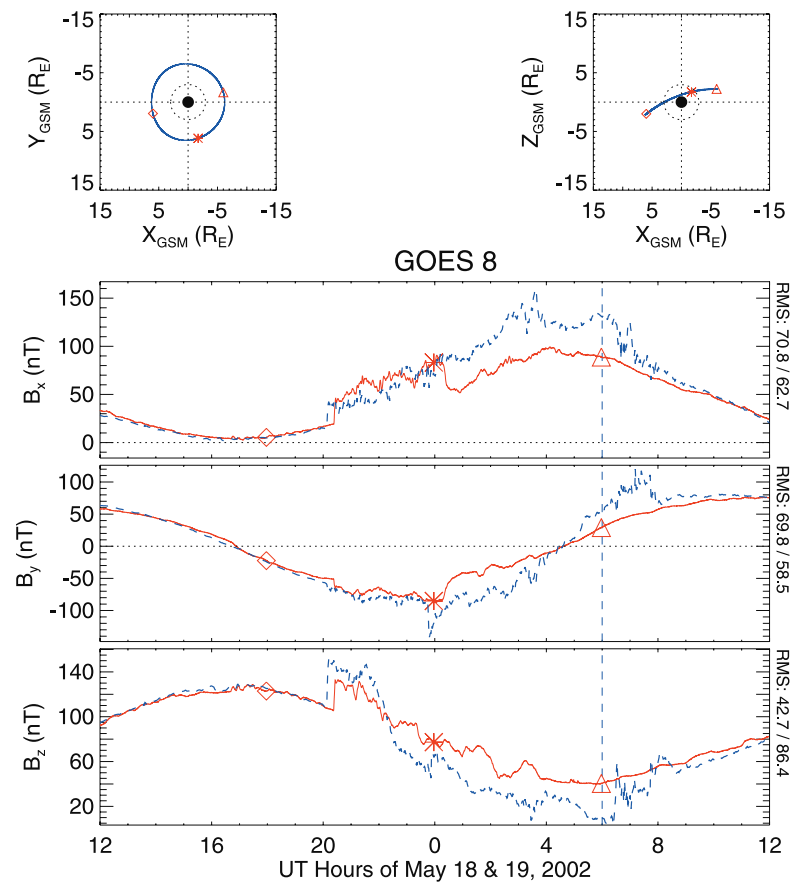
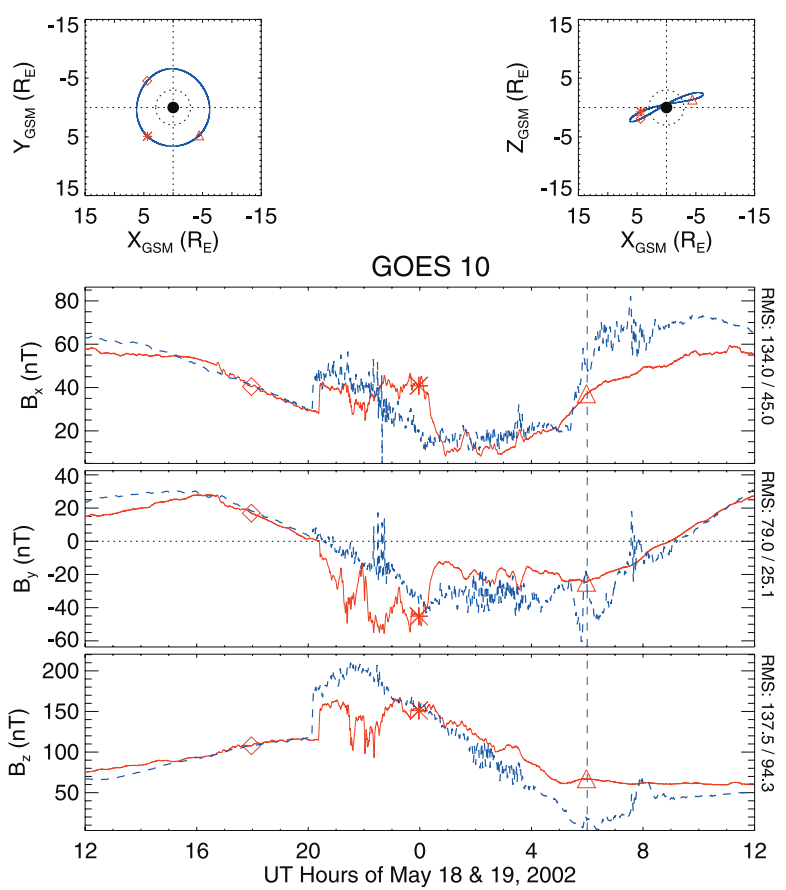

Figure 6. Same format as Figure 5, but for GOES 8 (left) and GOES 10 (right) with magnetic field components $\left(B_{x}, B_{y}\right.$, and $\left.B_{z}\right)$.
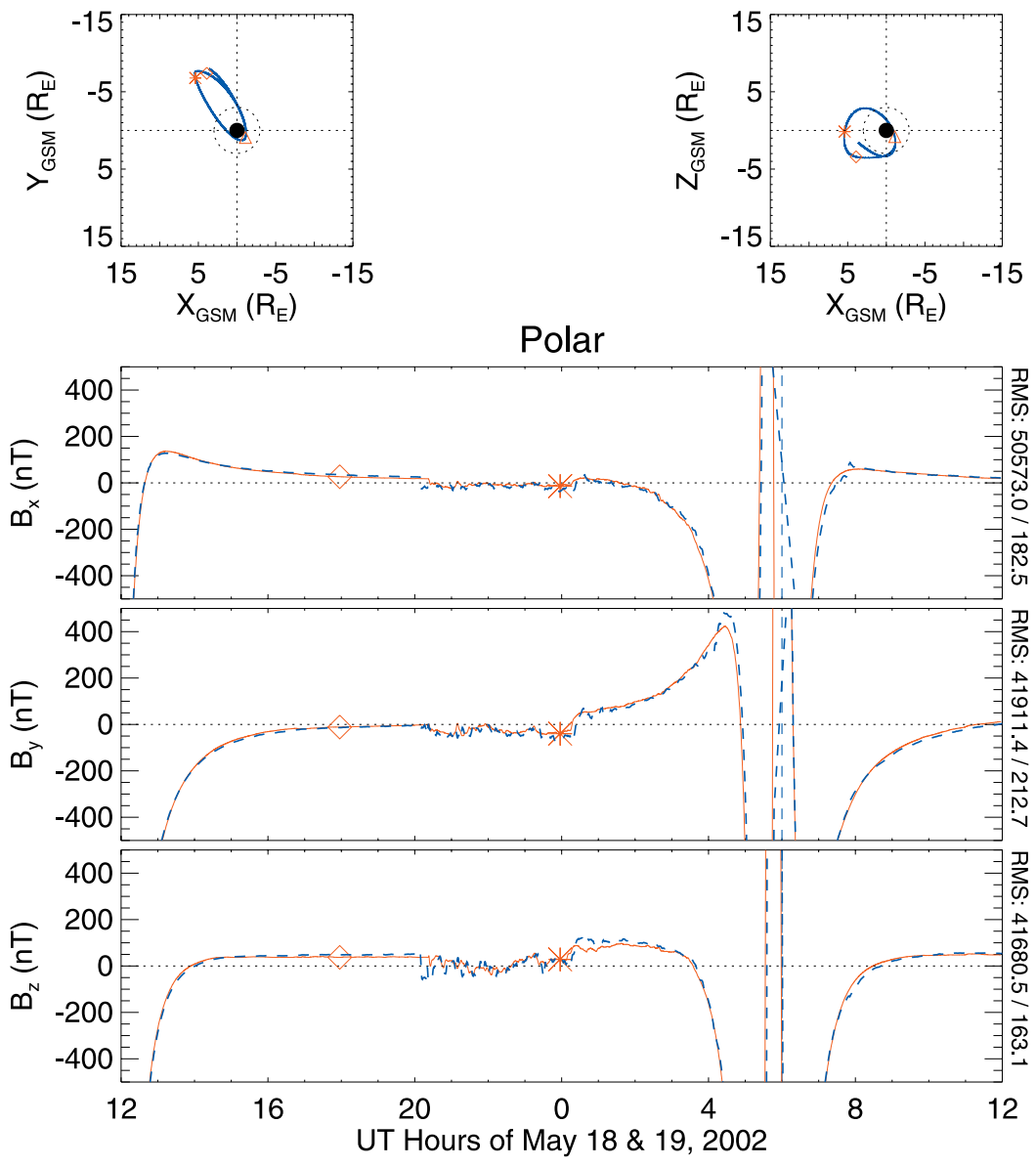

Figure 7. Same format as Figure 5, but for Polar with magnetic field components $\left(B_{x}, B_{y}\right.$, and $\left.B_{z}\right)$. 
top are the trajectories in the GSM-XY and GSM-XZ planes. Note that the $\mathrm{X}$ and $\mathrm{Y}$ axes are reversed to make the Sun to the left, dawnside at the top, and duskside at the bottom. The black solid circle shows the location and size of the Earth. The dotted circle denotes the inner boundary of the GM domain at $2.5 R_{E}$. A square, an asterisk, and a triangle are used to mark three sample data points both in the trajectory plots and in the following panels. The vertical dashed line marks the time point of the $D s t_{\min }$ of the storm. The root-mean-square (RMS) difference between measurements and simulated data, also called RMS error, and the RMS of measurements are indicated in the same units before and after the slash sign, respectively, on the right in each panel. The RMS error is used to quantitatively measure the simulation accuracy. The smaller the RMS error is, the better the performance of the model is.

[23] The magnetic field and plasma data from the Geotail spacecraft and the comparison with the model results are shown in Figure 5. From top to bottom are plotted magnetic field components $\left(B_{x}, B_{y}\right.$, and $\left.B_{z}\right)$, plasma number density $(n)$, and plasma velocity components $\left(V_{x}, V_{y}\right.$, and $\left.V_{z}\right)$. The magnetic field measurements are from the Magnetic Field Experiment (MGF) [Kokubun et al., 1994] and the plasma measurements from the Comprehensive Plasma Instrumentation (CPI) [Frank et al., 1994]. The measured magnetic field and plasma parameters in Figure 5 have similar variations to their counterparts in the solar wind input as shown in Figure 2, but with higher peak values except in $V_{x}$. These features show that Geotail was located around the bow shock, in the solar wind or in the magnetosheath during the storm. As shown in Figure 5, the observations and simulations generally agree quite well with each other. However, there are four notable discrepancies between them as well. First, the simulation does not capture several abrupt fluctuations observed by Geotail, e.g., those in the period of 0300-0400 UT on 19 May 2002. This inconsistency may be due to the limitations associated with the MHD approximations in the BATS-R-US. Also, considering the substantial $V_{y}$, another possible reason is that the actual solar wind hitting the magnetosphere had a structure that the solar wind going by the ACE did not have. Second, in the first 4 hours of the simulated 24 hours, the model results are more similar to the propagated ACE data than the Geotail observations. This implies that the bow shock in reality may be further away from the Earth than the simulated one, which makes Geotail be in the solar wind during the 4 hours in the simulation. Next, between 2015 UT on 18 May and 0420 UT on 19 May, the values of the simulated $n$ and $V_{z}$ are different from the measured ones but almost the same as the timeshifted ACE data. Even though the peak values in $B_{x}, B_{y}, V_{x}$, $V_{y}$, or $V_{z}$ have only a slight inconsistency between the simulation results and the observations, they are all much larger than those in the solar wind input. These signatures indicate that the simulated positions of Geotail should be still in the solar wind during the first simulated 16 hours but then be located in the modeled magnetosheath afterward to agree with the Geotail observations. Last, compared with the Geotail measurements, one can see a time lag of $\sim 15 \mathrm{~min}$ in the simulated plasma data, which is more apparent in the pulses of the density and velocity components at $\sim 2015$ on 18 May. Nevertheless, this is not the case in the modeled magnetic field. The time delay in plasma but not in magnetic field may be related to the limitations of the ACE data propagation method, the MVA technique [Weimer et al., 2003; Weimer, 2004] which gives emphasis to the IMF fluctuation tilt with regard to the Sun-Earth line during the propagation and ignores the interactions of IMF and plasma in the interplanetary medium.

[24] The magnetic field data from GOES 8 and GOES 10 and the comparison with the simulation results are shown on the left and right in Figure 6, respectively. Plasma data were not available from the two spacecrafts. Both GOES 8 and 10 are at geosynchronous orbit. The magnetic field measurements are reproduced reasonably well with excellent agreement with respect to overall trends, but there are several discrepancies in the details. Perturbations in the measured and simulated data for GOES 8 and 10 clearly show how the solar wind shock and sheath in front of the magnetic cloud, and the cloud itself impact the magnetic field at geostationary orbit. Both in the observations and in the simulation, $B_{x}$ and $B_{z}$ both increase suddenly at about 2015 UT on 18 May in response to the interplanetary shock hitting the magnetosphere. When the sheath and the leading field of the magnetic cloud passed by the magnetosphere, the geosynchronous-orbit magnetic field was disturbed from 2015 UT on 18 May to 0830 UT on 19 May, or in the initial phase, the main phase, and the early recovery phase of the storm. Consistent with the time delays in the simulated plasma parameters at Geotail, the modeled magnetic field components at GOES 8 and 10 lag the observations by a same period of $\sim 15 \mathrm{~min}$. RMS errors indicate that the measurements from GOES 10 are reproduced better than those from GOES 8; this is true even during the first 4 hours and last 2 hours in the simulation via visual inspection.

[25] The magnetic field data from Polar and the comparison with the simulation results are shown in Figure 7. In the simulated 24 hours, the Polar Magnetic Field Experiment (MFE) sampled the magnetic field in a wide range of radial distance, 1.5-9.6 $R_{E}$. The observed magnetic field magnitude is from 10.0 to $10,595.5 \mathrm{nT}$. The huge RMS errors are due to the fact that Polar stayed out of the computational domain for several hours but the modeled field values do not exceed those at the inner boundary. Note that the ranges of the three field components in Figure 7 are limited between -500.0 and $500.0 \mathrm{nT}$. The simulated data match the observed ones extremely well in the plot. Again, both of them begin to be disturbed at 2015 UT on 18 May when the interplanetary shock hits the magnetosphere. However, these disturbances end at 0020 UT on 19 May, not at a later time, 0830 UT on 19 May as seen in GOES 8 and 10. The reason is that starting at 0400 UT Polar moved so close to the Earth that the magnetic field is dominated by the dipole field and does not respond to the disturbances in the solar wind so obviously any more.

\subsubsection{LANL MPA}

[26] During the moderate storm, almost continuous plasma observations at geosynchronous orbit can be obtained from the MPA instruments onboard six of the seven Los Alamos National Laboratory (LANL) geostationary satellites operating at that time. 1989-046 is excluded because of large data gaps; the selected six satellites are 1990-095, 1991080, 1994-084, LANL-97A, LANL-01A, and LANL-02A. These satellites are located nearly in the geographic equatorial plane and make observations at different geographic 

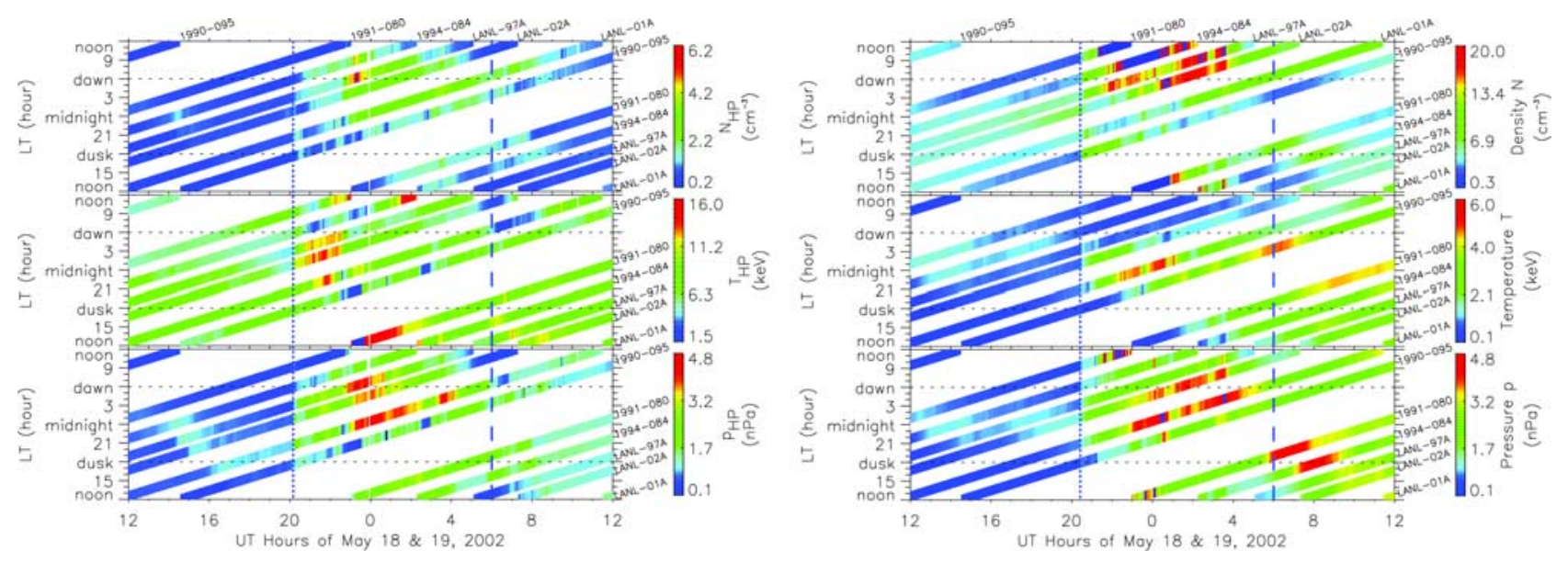

Figure 8. Local time (LT) versus universal time (UT) plots of LANL MPA plasma data (left) and corresponding simulation results (right) on the second half of 18 May and the first half of 19 May 2002. From top to bottom are color-coded number density $\left(N\right.$, in $\left.\mathrm{cm}^{-3}\right)$, temperature $(T$, in $\mathrm{keV})$, and pressure $(p$, in $\mathrm{nPa}$ ). Missing data are colored white, and black (purple) indicates that values are lower (higher) than the minimum (maximum) of corresponding color bar. The vertical dashed line indicates $D s t_{\mathrm{min}}$; the vertical dotted one shows the approximate arrival time of the solar wind shock. Between the two horizontal dotted lines is the nightside. The names of six LANL geostationary satellites are shown at the top and on the right.

longitudes. As the nightside geosynchronous orbit is an altitude $\left(6.62 R_{E}\right)$ at the transition from the plasma sheet in the magnetotail to the storm-time ring current, plasma observations from the MPA instruments are particularly useful in investigating the ring current development during disturbed times [e.g., Thomsen et al., 1998a, 2003; Denton et al., 2005, 2006; Zhang et al., 2006]. The detailed MPA design and its operating characteristics were described by Bame et al. [1993], and McComas et al. [1993] demonstrated the capabilities and typical observations of the MPA instruments for the first time.

[27] Because the MPA instruments measure only energy per charge and thus cannot distinguish the composition of positively charged particles, it is assumed that all ions are protons. Figure 8 shows number density $(N)$, temperature $(T)$, and pressure $\left(p=N K_{B} T\right.$, where $K_{B}$ is the Boltzmann constant) of the high-energy protons with a range from 0.1 to $45 \mathrm{keV}$, denoted by "HP," from the MPA (left) and the simulation results (right) from "virtual satellites," which fly through the GM/BATS-R-US output and extract the model results at the exact time and location of the six LANL satellites. Note that the simulation results for Geotail, GOES 8, GOES 10, and Polar are also obtained in the same way. From top to bottom in Figure 8, density, temperature, and pressure are color-coded in the LT versus UT plots, respectively. Missing data are colored white, and black (purple) indicates that values are lower (higher) than the minimum (maximum) of the corresponding color bar. Again, the vertical dashed line indicates the $D s t_{\min }$. The nightside is between the two horizontal dotted lines. The six LANL satellite names are shown at the top and on the right. The LT versus UT plots can also be called LT-UT maps which are a convenient method of displaying the large amounts of data. Since the LT-UT maps describe both the spatial and temporal variations of the plasma parameters at geosynchronous orbit, the timing and asymmetry of inten- sifications in the quantity are readily apparent on them [Zhang et al., 2006].

[28] As shown in both the MPA data and the simulation results displayed in Figure 8, plasma density, temperature, and pressure vary with both LT and UT. That is, each of them is a function of LT and UT. After the arrival of the interplanetary shock, all of the three parameters are disturbed. They display obvious enhancement regions and show distinct peaks. Similar to the simulated magnetic fields at geosynchronous orbit for GOES 8 and 10, all three parameters from the simulation lag the MPA measurements by the same $\sim 15 \mathrm{~min}$, which is shown most apparently in the pressure panels at the time when the shock hit the magnetosphere (indicated by two vertical dotted lines in Figure 8). The same time lags and simultaneous disturbances in the magnetic field and the plasma confirm that the coupling of the GM and IM components in the SWMF is self-consistent. Compared with previous studies in which the magnetic field in the magnetosphere is prescribed with a magnetic field model to usually be a dipole or a more realistic field [e.g., Fok and Moore, 1997; Kozyra et al., 2002; Jordanova et al., 2003; Chen et al., 2003], this is an important improvement in the simulation of the inner magnetosphere, especially with a global coupled code.

[29] Several discrepancies between the measurements and the simulation results also exist. Although the locations on the LT-UT maps (LT and UT times) and the peak values of the pressure enhancements observed by LANL-01A and LANL-02A are reproduced reasonably well, one can not find counterparts in the MPA data for the modeled pressure increases at around noon and in the premidnight sector on 1991-080 and 1994-084. Unlike that in the pressure panels, the ranges of the color bars in the density and temperature panels are different between the observations and the model results. Except that the peak locations slightly agree with each other at 0000 UT on 19 May, the values in density and 

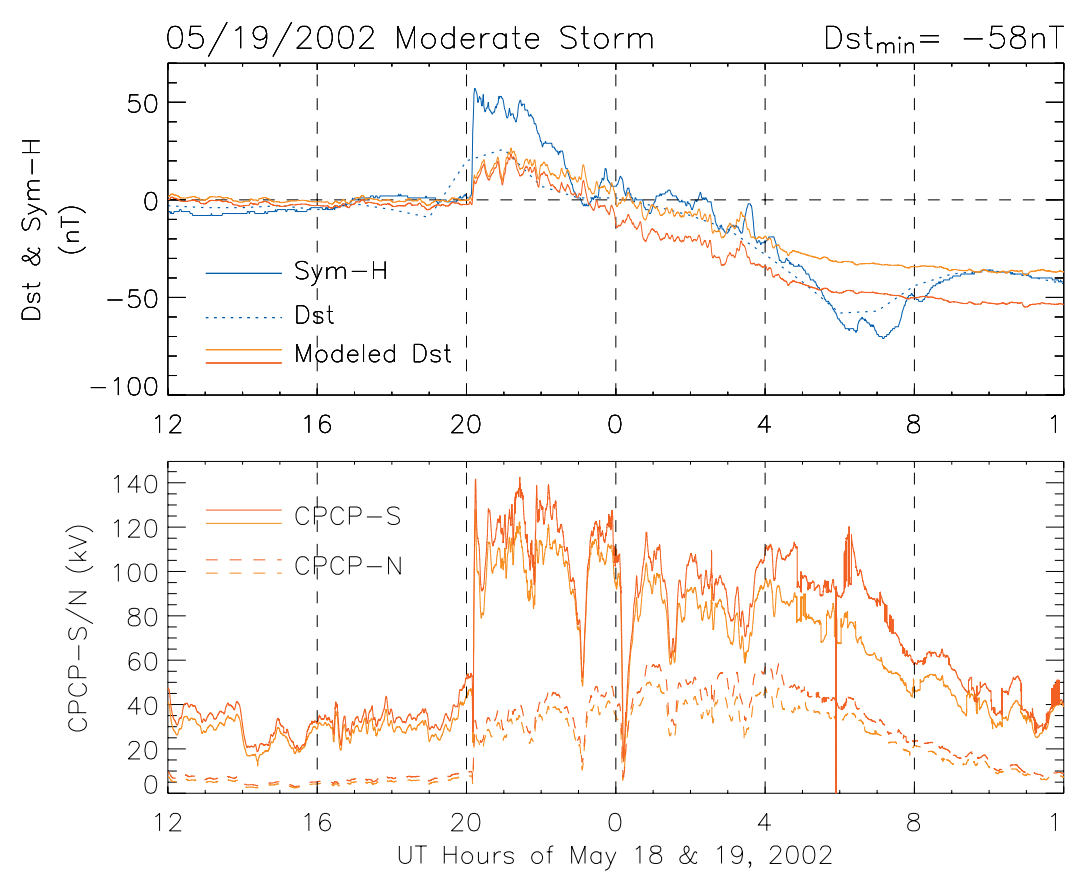

Figure 9. Observed 1-min Sym-H and 1-hour Dst indices (in blue), simulated Dst, CPCP-S and CPCP$\mathrm{N}$ (in yellow or red) in two runs on the second half of 18 May and the first half of 19 May 2002. The only difference between the two runs is the density at the inner boundary (@2.5 $R_{E}$ ) of the GM domain: yellow, $5 \mathrm{~cm}^{-3}$; red, $10 \mathrm{~cm}^{-3}$. See the text for details.

temperature in the simulation are not comparable to the MPA hot-proton measurements. In the run, a super dense but cold population of plasma is found at geosynchronous orbit. However, the simulated total plasma pressure represents the hot-proton pressure in the MPA data quite well.

[30] There are several reasons for the data-model differences. First, shown on the left of Figure 8 are only the hot-proton bulk properties. After checking the MPA measurements for the cold protons with energy less than $0.1 \mathrm{keV}$ (not shown here), one finds very dense, as high as $84 \mathrm{~cm}^{-3}$, but low-temperature protons near local noon, which have similar enhancements to those in the simulation. Nonetheless, the cold-proton MPA observations appear to be related to the plasmaspheric drainage plume, but the MHD results look like magnetosheath material that has entered the magnetosphere. In addition, the total pressure is carried mainly by the hot ions because of their relatively higher temperatures [Daglis et al., 1999, and references therein]. It is believed that this is the main reason for the agreement in the pressure MPA data-model comparison. Furthermore, the plasma properties in the plasma sheet at geosynchronous orbit are highly variable at widely different time scales [Thomsen et al., 1996, 1998b; Borovsky et al., 1998a, 1998b; Zhang et al., 2006]. Last, the drift paths of particles, especially ions, in the inner magnetosphere are complex. They depend on levels of geomagnetic activity, the first invariant (magnetic moments), and the second invariant. In turn, magnetic moments are determined by magnetic field magnitude and particles' kinetic energy perpendicular to the magnetic field; the second invariant also has a dependence on the particle energy and more complicated properties of the magnetic field configuration. Therefore it is still a challenge to simulate plasma parameter variations in the near-Earth plasma sheet.

\subsubsection{Sym-H, Dst, and CPCPs}

[31] Shown in the top panel of Figure 9 are measured Sym-H and Dst indices in blue and simulated Dst in yellow or red. Dst is a popularly used geomagnetic index, but it has a low resolution of 1 hour and is calculated on the basis of horizontal magnetic field measurements from four geomagnetic observatories located near the equator [Sugiura and Kamei, 1991]. Sym-H, the symmetric disturbance portion of the horizontal magnetic field component, is also used in the data-model comparison, because it has a 1-min resolution, showing more details of the geomagnetic disturbances, and is created from six observatories in the midlatitude region [Iyemori and Rao, 1996]. Although a recent statistical study by Wanliss and Showalter [2006] shows $S y m-H$ can be used as a high-resolution Dst, obvious discrepancies can be seen between them during this moderate storm, especially in the 3 hours before the SSC, in the initial phase and around $D s t_{\text {min }}$. In this storm event, the minimum $S y m-H$ even trails $D s t_{\min }$ by over 1 hour.

[32] The modeled Dst is obtained by the Biot-Savart formula

$$
\mathbf{B}=\frac{\mu_{0}}{4 \pi} \int \frac{I d \mathbf{l} \times \hat{\mathbf{r}}}{r^{2}}
$$

where $\mathbf{B}$ is the disturbed magnetic field, $\mu_{0}$ is the permeability constant, $I$ is the current, $d \mathbf{l}$ is the differential length vector of the current element, $\wedge^{r}=\mathbf{r} / r$ is the unit vector in the direction of $\mathbf{r}$, and $r$ is the distance from the current element to the field point. The magnetic field disturbance at the center of the Earth along the positive Z-axis in GSM coordinates is taken as a proxy of Dst. In the simulation, the Dst calculation is performed every four seconds for all the 
electric currents in the computational domain which include the ring current, magnetopause current, tail current, fieldaligned currents, etc. Modeled Dst indices in two runs are shown in Figure 9. The only difference between the two runs is the density at the inner boundary $\left(@ 2.5 R_{E}\right)$ of the GM domain: $5 \mathrm{~cm}^{-3}$ denoted by the yellow line and $10 \mathrm{~cm}^{-3}$ by the red line. Note that the density at the inner boundary is a proxy of outflow ions: the higher the inner boundary density, the heavier the ionospheric ion outflow. It is also noted that for the purpose of comparison the modeled Dst values from both the runs are shifted $\sim 15$ min earlier to make the simulated SSC appear at the same time as the sudden jump in Sym- $H$.

[33] As shown in Figure 9, in general, both the modeled Dst indices in the two runs (in red and yellow) agree reasonably well with the measured $S y m-H$ and Dst. After increasing the mass density at the inner boundary of the GM domain artificially by a factor of 2 (to a density at $2.5 R_{E}$ equal to $10 \mathrm{amu} / \mathrm{cm}^{3}$ ), one can see that Dst is then slightly lower than that with $5 \mathrm{amu} / \mathrm{cm}^{3}$ at $2.5 R_{E}$ before $2200 \mathrm{UT}$ on 18 May, but the difference, up to $20 \mathrm{nT}$, is obvious afterward. This result indicates that low-altitude (at $2.5 R_{E}$ ) plasma conditions can play an important role in the development of a storm.

[34] Like the measured Dst, the modeled Dst indices are clearly different from the measured $S y m-H$ in the initial phase. In the main phase, one can see a 15-min time shift between modeled Dst and Sym-H, which can be found easily near the variation spikes at 0300-0400 UT on 19 May. This time inconsistency may be related to the error in the MVA technique which was used to propagate the solar wind data. Compared to measurements, a big discrepancy can also be found in the modeled Dst in the late main phase. It is believed that the lack of outflow of heavy ions (mainly $\mathrm{O}^{+}$) from the ionosphere in the simulation is the main factor (the BATS-R-US assumes that the simulated ions are all $\mathrm{H}^{+}$), causing the simulated $D s t$ values not to diminish as sharply as in reality [e.g., Kremser et al., 1987; Hamilton et al., 1988; Daglis et al., 1999, and references therein]. Because no losses except for the large-scale magnetospheric convection are considered in the simulation, the simulated storm does not have a recovery phase and the developed ring current does not fade away. In previous studies [e.g., Kistler et al., 1989; Fok et al., 1991; Jordanova et al., 1996; Kozyra et al., 1998a], it is found that in the ring current charge exchange dominates other ion loss processes, such as precipitation into the atmosphere, wave-particle interaction, and Coulomb collisions. This classic view is confirmed to be accurate in the storm simulation with a missing recovery phase due to no charge exchange ion loss in the RCM.

[35] Modeled cross polar cap potentials in the Southern and Northern Hemispheres, or CPCP-S and CPCP-N, respectively, are plotted in the bottom of Figure 9. CPCP-S is marked by the solid lines and CPCP-N by the dashed ones. Results with the inner boundary mass density equal to 5 (10) $\mathrm{amu} / \mathrm{cm}^{3}$ are denoted by the yellow (red) lines. Like the modeled Dst, all CPCPs, CPCP-S or CPCP-N, are also shifted $\sim 15$ min earlier than the actual times from the simulation. As seen in Figure 9, the simulated CPCP-S and CPCP-N, which are in reasonable agreement with those in previous studies [Nopper and Carovillano, 1978; Boyle et al., 1997; Weimer, 1995, 2001; Siscoe et al., 2002; Ober et al., 2003; Rothwell and Jasperse, 2006], respond well to the disturbances in the solar wind. In both runs, CPCP-N and CPCP-S are almost tripled in a short time at the SSC; they stay disturbed and enhanced in the initial and main phases, and they begin to recover to the prestorm levels with the start of the recovery phase. In addition, the modeled CPCPs also show dependence on the density at the inner boundary layer. That is, CPCPs in the run with higher density at the inner boundary $\left(2.5 R_{E}\right)$ are generally larger than those with lower density at $2.5 R_{E}$. Another interesting feature in the CPCPs is that CPCP-S is almost always significantly larger than CPCP-N either at quiet times or at active times; in the two runs, CPCP-S peaks at $141.7 \mathrm{kV}$, but the CPCP-N peak value is only $59.5 \mathrm{kV}$. The difference between CPCP-S and CPCP$\mathrm{N}$ is mainly due to the tilt of the Earth's dipole, which in turn causes the solar EUV fluxes and then the solar-EUV-generated ionospheric conductance in the IE component to be different from each other in the two hemispheres [Ridley et al., 2004]. In test simulations, it is found that CPCP-N is significantly larger than CPCP-S during storms that occur in Northern Hemisphere winter because the solar EUV flux is higher in the Southern Hemisphere than Northern at that time of year. Thus it is certain that the modeled CPCPs in the two hemispheres have a seasonal distribution. These CPCP features are in some agreement with the results from observation-based models. For instance, it was found in the DMSP-based ionospheric convection model (DICM) [Papitashvili and Rich, 2002] that CPCPs do have seasonal dependence, but it is not as significant as in the SWMF simulation; the results from the Assimilative Mapping of Ionospheric Electrodynamics (AMIE) technique [Richmond and Kamide, 1988] showed that the CPCPs in the two hemispheres are different. Again, the difference was not that large and observed only under particular conditions: $B_{z}>0$ and $\left|B_{y}\right|>B_{z}$, in which $B_{y}$ plays a dominant role in controlling the convection patterns in the high-latitude ionosphere [Lu et al., 1994; G. Lu, private communication, 2005]. It is also found that CPCPs from the AMIE (not shown here) are between the simulated CPCP-S and CPCP$\mathrm{N}$ shown in Figure 9.

\subsection{Activity in the Inner Magnetosphere}

[36] Figure 10 shows snapshots of the modeled plasma mass density $(\rho)$, temperature $(T)$, and pressure $(p)$ from the GM/BATS-R-US component in the GSM-XY plane at the SSC, and 3, 6, 9, and 12 hours after the SSC. Values lower (higher) than the minimum (maximum) of corresponding color bar are colored blue (red). Each snapshot shows dayside to the left, nightside to the right, dawn at the top, and dusk at the bottom. On every snapshot, geostationary orbit is projected onto the GSM-XY plane and denoted approximately by the solid grey circle. In the middle row (showing $T$ ), the outer boundary of each snapshot indicates the lowest altitude of the open-closed boundary of the magnetic field in the simulation. Note that the border of the open and closed field lines in the GSM-XY plane is not a circle or ellipse [De Zeeuw et al., 2004]. The plot at the top of Figure 10 is in the same format as Figure 9 but showing only observed $S y m-H$ and $D s t$ indices and modeled $D s t$ in the run with the density at the inner boundary equal to $10 \mathrm{~cm}^{-3}$. 


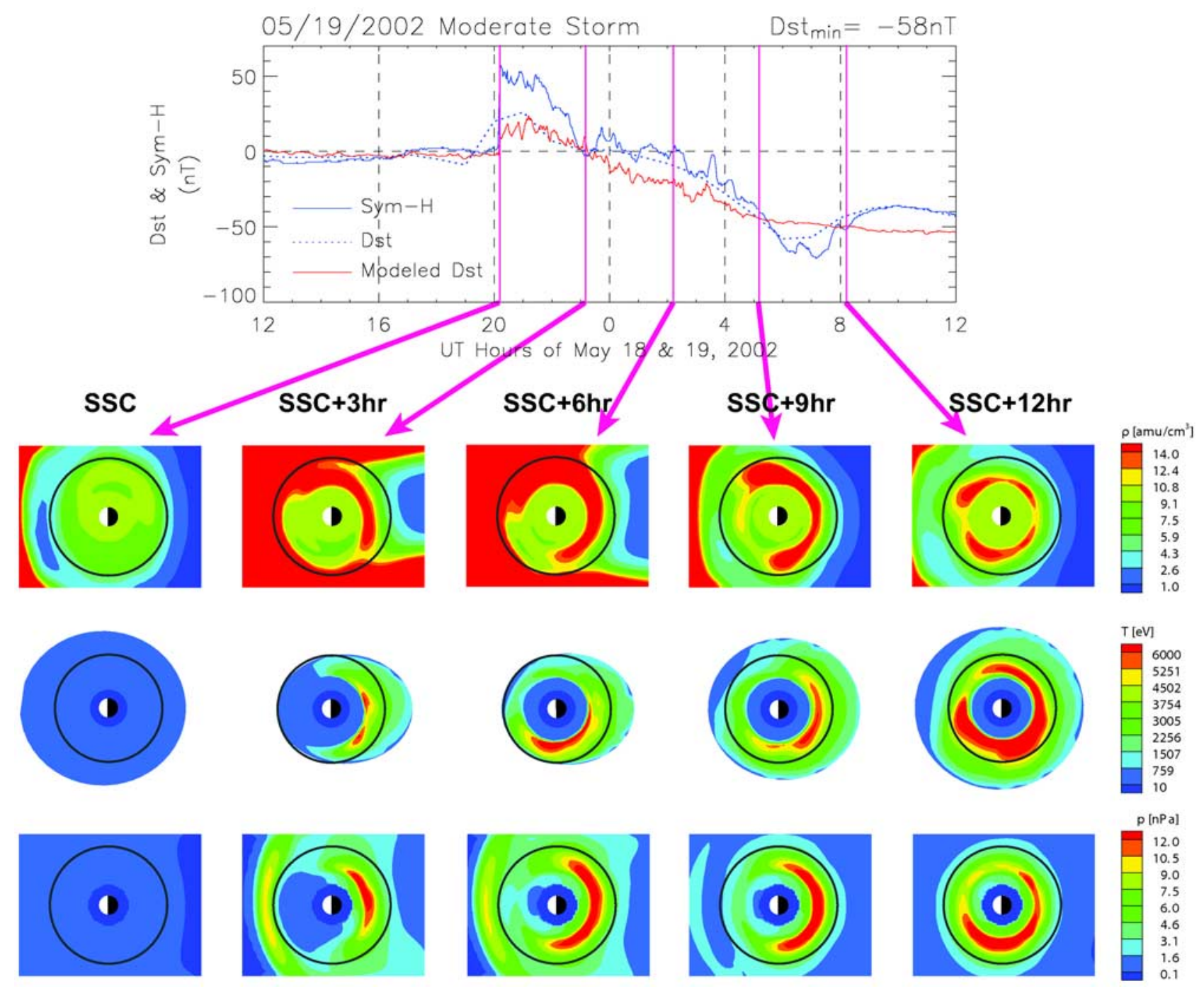

Figure 10. Snapshots of the simulated plasma mass density $\left(\rho\right.$, in amu $\left./ \mathrm{cm}^{3}\right)$, temperature $(T$, in $\mathrm{eV})$, and pressure $(p$, in $\mathrm{nPa}$ ) in the GSM-XY plane at five time points: the Sudden Storm Commencement (SSC), and 3 hours, 6 hours, 9 hours, and 12 hours after the SSC. On each snapshot, dayside (nightside) is on the left (right) and dawn (dusk) is at the top (bottom); the projection of the geostationary orbit onto the GSM$\mathrm{XY}$ plane is denoted approximately by the solid circle; the Earth is shown by a white/black round region. The figure at the top is in the same format as Figure 9, but showing only the top panel for the run with the inner boundary density equal to $10 \mathrm{~cm}^{-3}$.

[37] Like the geomagnetic indices, the three plasma parameters $(\rho, T$, and $p)$ as well as the open-closed boundary of the magnetic field respond well to the solar wind disturbances during the moderate storm. Three hours after the shock ahead of the MC hits the magnetosphere, a population of dense and cold magnetosheath-like plasma has access to a majority of geosynchronous orbit (except for the nightside part with LT ranging from $\sim 1900$ to $\sim 0500$ ); it fills the entire dayside magnetosphere and the dawn and dusk flanks of the magnetosphere. Note that the solar wind shock wave is extremely strong and pushes the dayside magnetopause inward to an altitude as low as that of the geostationary orbit.

[38] Like the hot-ion LT asymmetries as shown in Figure 1, $\rho$ near dawn is higher than around dusk in the ring current region which is similar to the result from a 3-D kinetic model by Nishikawa [1997]; $T$ is enhanced more obviously in the premidnight sector and right after midnight but $p$ is higher on the nightside and the enhancement region of $p$ displays a near dawn-dusk symmetry (more enhanced in the postmidnight sector) on the nightside as well as in the magnetosheath. The locations of the dayside magnetopause, which can be identified more easily in the $p$ snapshots, agree well with the model results by Shue et al [1998]. At 6,9 , and 12 hours after the SSC, $\rho$ peaks to values as high as $135.7 \mathrm{amu} / \mathrm{cm}^{3}$ in the magnetosheath and its enhancement in the partial ring current continues to exhibit a dawn-dusk asymmetry and spreads out in local time. Plasmas are hotter in the afternoon and premidnight sectors with the $T$ peak value equal to $10.0 \mathrm{keV}$. Pressure is enhanced to a peak value of $16.7 \mathrm{nPa}$ in the late main phase. In addition, the $p$ enhancement region is still generally nightside-symmetric 


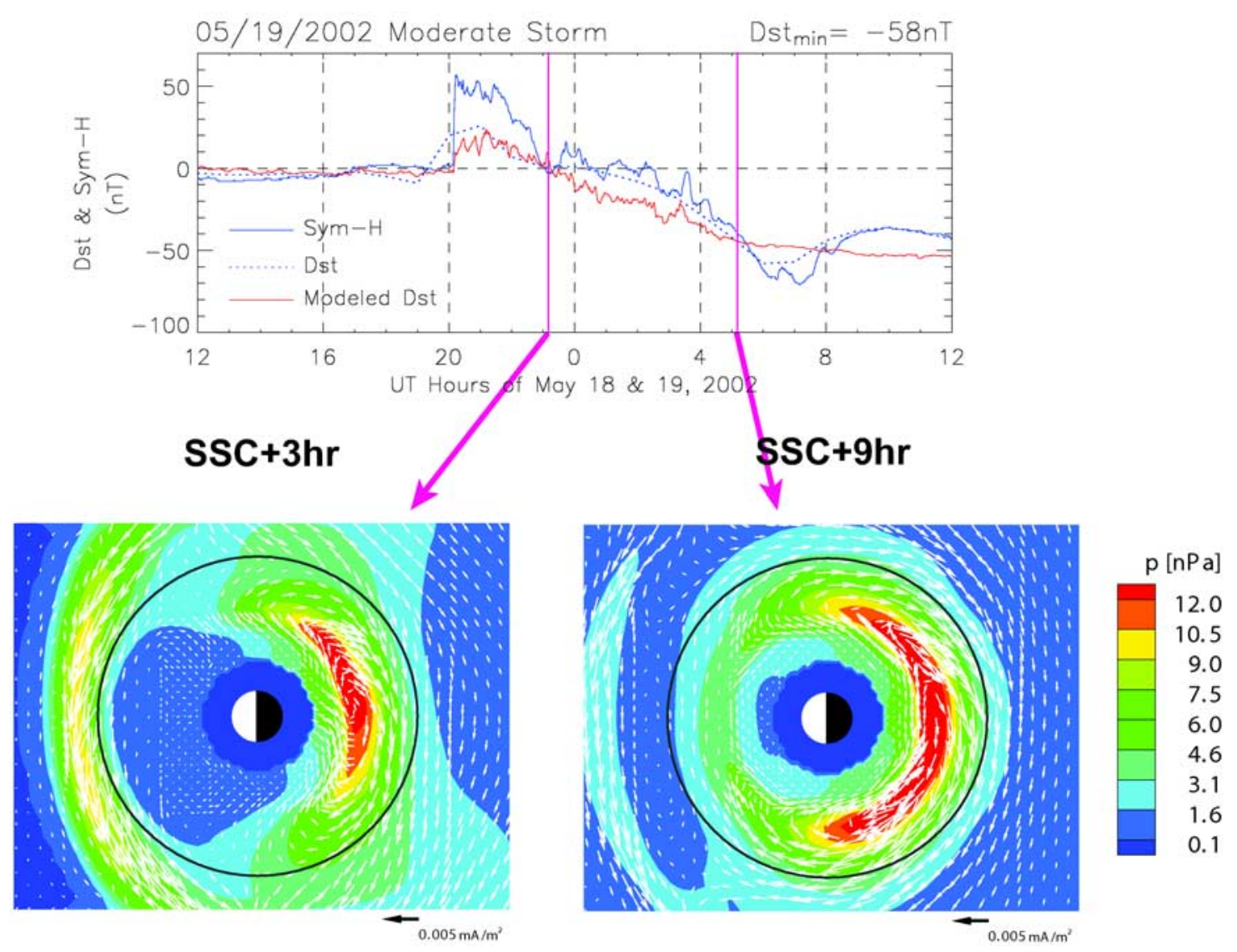

Figure 11. Same format as Figure 10, but showing only two pressure snapshots at 3 and 9 hours after the SSC. The color-coded pressure is overlapped with the magnitude and direction of the current density which are indicated by white arrows. A black reference vector, indicating a magnitude of $0.005 \mathrm{~mA} / \mathrm{m}^{2}$, is also shown below each snapshot on the right.

before $D s t_{\min }$, but it moves westward in the early recovery phase because no fresh particles are injected into the inner magnetosphere. The local time characteristics that $\rho$, $T$, and $p$ exhibit at geosynchronous orbit in the simulation are well in agreement with those in the MPA statistical study by Zhang et al. [2006].

[39] Although the dawn-dusk asymmetries in density and temperature in the simulated ring current $\left(<6.62 R_{E}\right)$ are almost the same as those of geosynchronous orbit hot ions in Figure 1, it is found that the modeled ring current plasmas are much denser and colder than expected, e.g., temperature in the ring current at quiet time is observed to be as high as $200 \mathrm{keV}$ during quiet time and decrease to be about tens of $\mathrm{keV}$ during storm time because newly injected plasma sheet-like ions dominate the ring current during the disturbed time [e.g., Daglis et al., 1999, and references therein]. This issue also exists in the simulated geosynchronous orbit plasmas as illustrated in Figure 8. However, the LT dependence and peak values of the plasma pressure are reproduced pretty well in the regions of geosynchronous orbit (see Figure 8).

[40] Figure 11 shows close-up views of the two pressure snapshots at 3 and 9 hours after the SSC. The current density vectors are overlapped over the color-coded pressure with their magnitude and direction indicated by those white arrows. A black reference vector, indicating a mag- nitude of $0.005 \mathrm{~mA} / \mathrm{m}^{2}$, is also shown below each snapshot on the right. The two snapshots show that the magnetospheric currents, such as the ring current, cross-tail current, and the dayside magnetopause current, are simulated successfully. With a reasonable peak value of $9.2 \mathrm{nA} / \mathrm{m}^{2}$ [e.g., Lui et al., 1987], the ring current is still partial or asymmetric because it is closed through the region 2 field-aligned currents via the ionosphere [Iijima and Potemra, 1976]. In Figure 11, one can see that the partial ring current flows along constant- $p$ contours centered around the local $p$ peak. The pressure gradient in the inner part is steeper than in the outer part of the pressure peak. Consequently, the pressure gradient in the inner part (carrying the eastward current) is comparable to that in the outer part (carrying the westward current). Since the volume per unit $\mathrm{L}$ in the inner part is much smaller than that in the outer part, the net ring current flows westward [e.g., Ebihara and Ejiri, 2000]. The agreement between the current and the pressure patterns verifies that the MHD code, GM, has found an approximate force equilibrium in the inner magnetosphere, since the current equation comes directly from the MHD momentum equation if inertial terms are neglected. Eventually, the partial ring current evolves into a symmetric ring current in the late main phase; the enhancement regions of $\rho, T$, and $p$ display a symmetric distribution in the ring current. 


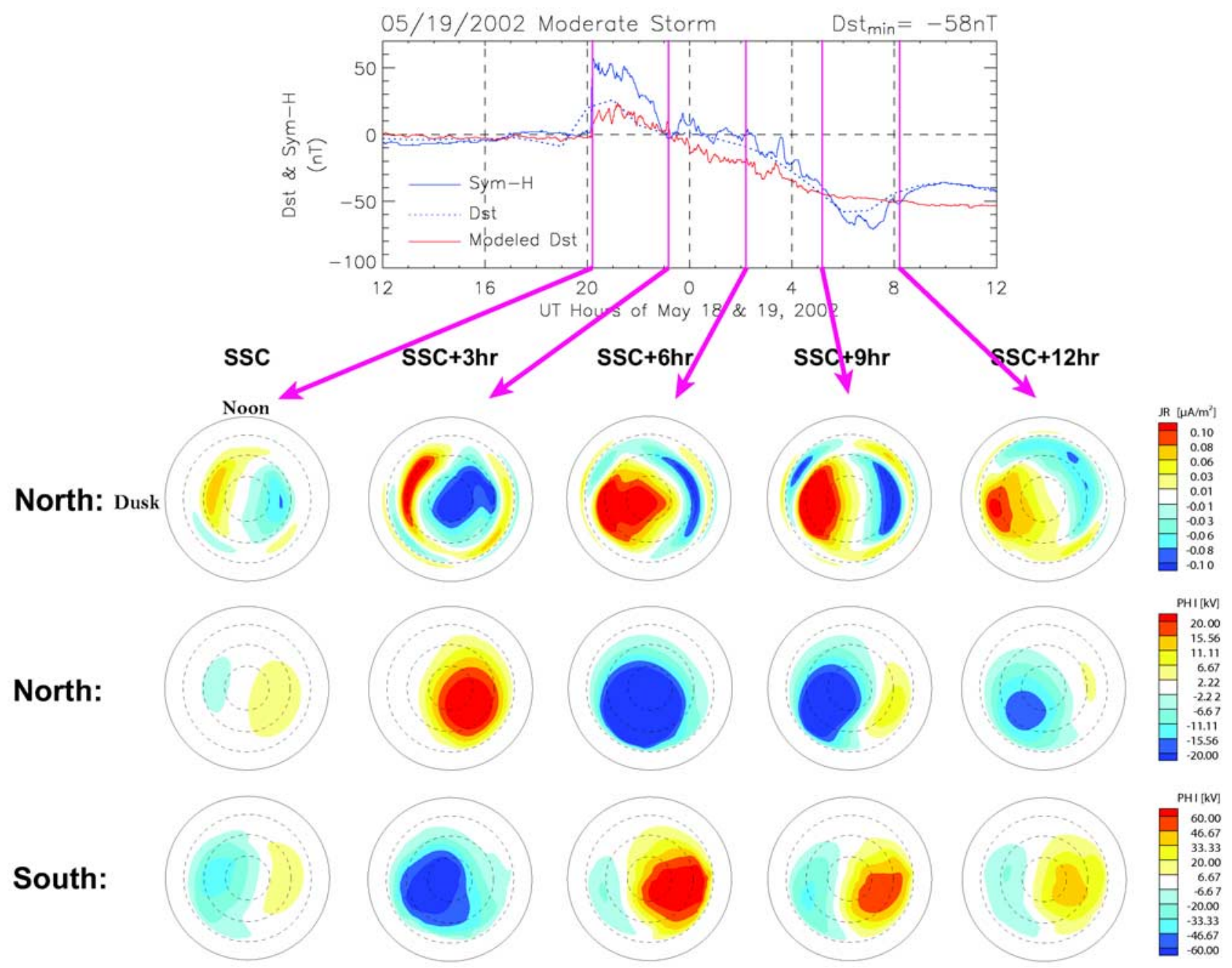

Figure 12. Same format as Figure 10, but for the simulated activity in the polar regions. In the top two rows are the field-aligned currents $\left(J R\right.$, in $\left.\mu \mathrm{A} / \mathrm{m}^{2}\right)$ and the corresponding electric potential $(P H I$, in $\mathrm{kV})$ in the Northern Hemisphere; for comparison, the electric potential $(P H I$, in $\mathrm{kV})$ in the Southern Hemisphere is plotted in the bottom row. Each snapshot shows noon (midnight) at the top (bottom), and dusk (dawn) to the left (right) with the lowest magnetic latitude plotted at $50^{\circ}$.

\subsection{Activity in the Polar Regions}

[41] Figure 12 shows the field-aligned current patterns in the Northern Hemisphere (top row) and the electric potential patterns in the Northern and Southern Hemispheres (middle and bottom rows) at the SSC, and 3, 6, 9, and 12 hours after the SSC. Again, values lower (higher) than the minimum (maximum) of corresponding color bar are colored blue (red). All snapshots are plotted in magnetic latitude-magnetic local time coordinates, with noon at the top and dawn to the right. The outer circle, or the lowest plotted magnetic latitude, is $50^{\circ}$ and the other rings mark $10^{\circ}$ latitude increments. In the top row, positive (negative) values indicate that currents are downward (upward), i.e., flowing into (out of) the polar region.

[42] In general, activity in the high-latitude ionosphere is produced reasonably well; the simulated patterns of the ionospheric potential and field-aligned currents are consistent with statistically obtained ones [Iijima and Potemra, 1976]. Compared to the one at the SSC, the region 1 fieldaligned current is highly enhanced 3, 6, and 9 hours after the
SSC. Meanwhile, consistent with the ring current development, the region 2 current begins to be enhanced, while it is almost always weaker than the region 1 current. Nine hours after the SSC, the region 1 current develops a classic distribution at high latitudes during the storm times [Iijima and Potemra, 1976; Waters et al., 2001; Papitashvili et al., 2002] with the region 2 current stronger on the dayside. However, one can see an additional equatorward current system around the duskside and dawnside, which is more obvious 3, 6, and 9 hours after the SSC. This additional current system is also a region 2 one; it is associated with the inner partial ring current which flows eastward as shown Figure 11. In the recovery phase which can be identified in the observed Dst, all the high-latitude ionospheric currents in the simulation are found to decay as expected. Note that the potential patterns shown in the middle and bottom rows of Figure 12 are on different scales: -20 to $20 \mathrm{kV}$ versus -60 to $60 \mathrm{kV}$. Thus the potential magnitudes in the Northern and Southern Hemispheres are quite different as also shown in Figure 9. Moreover, except for those at the 
SSC and 9 hours after the SSC, the potential patterns in the two hemispheres show distinct differences from each other. However, the asymmetric distribution of the cells on the dawnside and duskside can be readily noticed on the snapshots at 3 and 6 hours after the SSC. The main reason for this feature is that the IMF $B_{y}$ component is strong during this time period: $-16.6 \mathrm{nT}$ and $12.1 \mathrm{nT}$ at the two time points, respectively (see Figure 2). As reported in previous studies [Heppner, 1972; Taguchi et al., 1993; Lu et al., 1994; Papitashvili et al., 2002; Lukianova and Christiansen, 2006], a strong IMF $B_{y}$ produces asymmetric convection between the northern and southern polar caps and an interhemispheric asymmetry with regard to the noon meridian in the potential and field-aligned current patterns. In the Northern Hemisphere, the positive dawn potential cell is enhanced for negative IMF $B_{y}$ at $\mathrm{t}=\mathrm{SSC}+3$ hours. Three hours later at SSC +6 hours, as the IMF $B_{y}$ becomes strong and positive the negative dusk potential cell becomes enhanced. The opposite effect is seen in the Southern Hemisphere.

\section{Discussion}

[43] Using the coupled GM, IM, and IE components of the newly developed SWMF, a successful global simulation was performed of the moderate storm on 19 May 2002. Considering the model limitations such as the MHD approximations, almost all observed values are reproduced reasonably well both in the pre-storm quiet times and in the moderately disturbed times. In other words, the SWMF is reaching a level of sophistication for allowing quantitative comparison with observations. This has important impacts on and applications in space weather studies, such as understanding storm physics and predicting storms through simulations which are conducted faster than real time on massively parallel supercomputers. Furthermore, the simulation results in this study can reveal some problems in the current SWMF, e.g., the dense but cold plasmas in the inner magnetosphere in the simulation. They also provide some clues about how to improve the SWMF performance to make a better simulation of the Earth's magnetosphere and its coupling with the ionosphere, especially during storm times.

[44] In the simulation, three different algorithms, BATSR-US, RCM, and RIM are shown to be successfully selfconsistently coupled. The achievement presented in this study is significant; it represents a benchmark for the state of progress in modeling inner magnetospheric plasmas with a global numerical code. This is the first paper in which a model, RCM, that represents ring current injection with selfconsistently computed electric and magnetic fields is actually compared with observations. The recovery phase is not reproduced because charge exchange is not considered in the RCM for the runs presented, which yet implies that charge exchange may be the dominant loss process in the ring current during storm recovery.

[45] It is shown in the simulation that activity in the global magnetosphere (including the outer and inner magnetospheres) and the ionosphere responds well to the changes in the solar wind and IMF. The development of the ring current during the storm is mainly controlled by the upstream solar wind conditions. In other words, it is confirmed in this global modeling that the upstream solar wind/IMF conditions control the storm activity and an important driver of the ring current intensification is the solar wind or interplanetary medium.

[46] The similarities of the simulation results to the superposed MPA measurements [Zhang et al., 2006] imply that the ring current source populations are beyond geosynchronous orbit in the plasma sheet [e.g., Smith et al., 1979; Chen et al., 1994; Kozyra et al., 1998b; Korth et al., 1999; Ebihara et al., 2005; Denton et al., 2005, 2006]. Before entering in the ring current region, the source particles move closer to the inner magnetosphere via their drift paths and have access to geosynchronous orbit. The drift paths, however, are dependent on species (ions or electrons), levels of geomagnetic activity, and adiabatic invariants of the particles. As found in previous studies [e.g., Korth et al., 1999; Korth and Thomsen, 2001; Ebihara and Fok, 2004; Zhang et al., 2006], the fluxes of particles with various energy levels have strong LT dependence in the inner magnetosphere. Consequently, the corresponding bulk plasma properties exhibit obvious asymmetries in the equatorial plane [Nishikawa, 1997; Korth et al., 1999; Denton et al., 2005; Zhang et al., 2006]: density is more enhanced around dawn; temperature is higher in the afternoon and premidnight sectors but enhanced plasma pressure is approximately symmetric around midnight on the nightside during the ring current development. However, as discussed in the study by Zhang et al. [2006], the drift paths are not the only reason for the density and temperature asymmetries. For instance, plasma transport via the low-latitude boundary layer (LLBL) into the magnetosphere may be another cause of the asymmetries. Solar wind plasma, first trapped in the magnetosheath, can enter the plasma sheet through both the high-latitude lobes and the LLBL. It has been found that the portion of plasma entering via the LLBL is both dense and cold [e.g., Eastman et al., 1976; Song and Russell, 1992, Fujimoto et al., 1996, 1998a, 1998b]. This cold, dense plasma, which has the characteristics of the magnetosheath, is found to be present near dawn both in the simulated ring current and in the superposed storm-time MPA observations at geosynchronous orbit [Zhang et al., 2006].

[47] Besides the solar wind entry, ionospheric outflow is also an important ring current source, especially during geomagnetic disturbed times. During storms, some cold ionospheric electrons and ions can first escape into the plasmasphere, plasma sheet, and magnetotail lobes [Lennartsson, 1997; Fuselier et al., 2003, and references therein] and then be energized and injected into the ring current region because of the enhanced magnetospheric dawn-dusk electric field. Note that there is no plasmasphere in the current SWMF. The ion outflow from the ionosphere and its impacts on the magnetosphere are modeled through a uniform source of ions at the high-latitude inner boundary (at $2.5 R_{E}$ ). In the quiet times before the storm and in the initial phase of the storm, the magnetospheric state and the intensity of the ring current are not much affected by the inner boundary density. The reason is that during northward IMF the ionsopheric plasma tends to populate in the lobe [Winglee, 1998]. However, in the main phase, the ionosphere-originated plasma can reach the inner magnetosphere under southward IMF [Winglee, 1998]. As a result, the density at the inner boundary can affect the ring current 
development. That is, a more intensified ring current is developed in the run with higher density at $2.5 R_{E}$. However, in the two runs, the simulated ring currents are not as intense as that observed in the late main phase, because the current SWMF assumes only a single ion species, protons, and no oxygen ions originate from the ionosphere. In spite of these modeling limitations, it is indicated indirectly that ionospheric ions, e.g., protons and oxygen ions, are important sources of the storm-time ring current particularly in the main phase. Moreover, in the late main phase, the outflow of oxygen ions may be even more substantial than the solar wind source in the development of the ring current. However, it should also be noted that according to the in situ measurements, the oxygen ions originated from the ionosphere do not always contribute significantly to the ring current especially during moderate storms [Daglis, 1997].

\section{Summary}

[48] The results from the data-model comparisons of the 19 May 2002 moderate storm are summarized as follows:

[49] 1. The SWMF is reaching a level of sophistication for accurately conducting storm simulations and allowing quantitative data-model comparisons both in the quiet times and active times in the global magnetosphere.

[50] 2. Some major storm characteristics are successfully reproduced, except for those in the recovery phase.

[51] 3. Because of the strong shock wave ahead of the magnetic cloud, in the initial phase and the early main phase, a population of dense and cold plasmas has access to most of the inner magnetosphere, except a region on the nightside.

[52] 4. Similar to those features of the superposed MPA hot-ion bulk properties at geosynchronous orbit [Zhang et al., 2006], the plasma parameters exhibit obvious dawndusk asymmetries or symmetries in the simulated ring current region: higher density near dawn, higher temperature in the afternoon and premidnight sectors, but almost symmetrically enhanced pressure on the nightside.

[53] 5. It is confirmed in this global modeling that the upstream solar wind/IMF conditions control the storm activity and an important plasma source of the ring current is in the solar wind. However, ionospheric outflow can also affect the ring current development, especially in the main phase. In the late main phase, the outflow of oxygen ions may play a key role in the ring current buildup.

[54] 6. Activity in the high-latitude ionosphere is produced reasonably well, but CPCP-S is almost always significantly larger than CPCP-N during the May storm, consistent with the seasonal dependence of ionospheric conductance.

[55] Acknowledgments. Magnetic field and plasma measurements from the ACE, Geotail, GOES 8 and 10, and Polar were obtained through the NSSDC in NASA Goddard Space Flight Center. James Weygand at UCLA helped us with the ACE data propagation. The WDC-C2, Kyoto provided the Dst and $S y m-H$ indices. Other MPA team members helped prepare the MPA data. This work was supported by NSF under grant numbers ATM-0402163 and ATM-0325255 and by NASA under grant numbers NNG05GJ89G and NNG05GM48G. The work of M. F. Thomsen and J. E. Borovsky at LANL was carried out under the auspices of the U. S. Department of Energy, with partial support from the NASA LWS program and an IGPP minigrant, under subcontract to the University of Michigan.

[56] Wolfgang Baumjohann thanks Yusuke Ebihara and George Siscoe for their assistance in evaluating this paper.

\section{References}

Bame, S. J., D. J. McComas, M. F. Thomsen, B. L. Barraclough, R. C. Elphic, and J. P. Glore (1993), Magnetospheric plasma analyzer for spacecraft with constrained resources, Rev. Sci. Instrum., 64, 1026.

Borovsky, J. E., M. F. Thomsen, D. J. McComas, T. E. Cayton, and D. J. Knipp (1997), Magnetospheric dynamics and mass flow during the November 1993 storm, J. Geophys. Res., 103, 26,373.

Borovsky, J. E., M. F. Thomsen, and R. C. Elphic (1998a), The driving of the plasma sheet by the solar wind, J. Geophys. Res., 103, 17,617.

Borovsky, J. E., M. F. Thomsen, R. C. Elphic, T. E. Cayton, and D. J. McComas (1998b), The transport of plasma sheet material from the distant tail to geosynchronous orbit, J. Geophys. Res., 103, 20,297.

Boyle, C. B., P. H. Reiff, and M. R. Hairston (1997), Empirical polar cap potentials, J. Geophys. Res., 102, 111.

Burlaga, L. F. (1991), Magnetic clouds, in Physics of the Inner Heliosphere, vol. 2, edited by R. Schwenn and E. Marsch, p. 1, Springer, New York. Chen, M. W., L. R. Lyons, and M. Schulz (1994), Simulations of phase space distributions of storm time proton ring current, J. Geophys. Res., 99, 5745

Chen, M. W., M. Schulz, G. Lu, and L. R. Lyons (2003), Quasi-steady drift paths in a model magnetosphere with AMIE electric field: Implications for ring current formation, J. Geophys. Res., 108(A5), 1180, doi:10.1029/ 2002JA009584.

Daglis, I. A. (1997), The role of magnetospohere-ionosphere coupling in magnetic storm dynamics, in Magnetic Storms, Geophys. Monogr. Ser, vol. 98 , edited by B. T. Tsurutani et al., pp. 107-116, AGU, Washington, D. C.

Daglis, I. A., R. M. Thorne, W. Baumjohann, and S. Orsini (1999), The terrestrial ring current: Origin, formation, and decay, Rev. Geophys., 37, 407.

Delamere, P. A., D. W. Swift, and H. C. Stenbaek-Nielsen (2001), An explanation of the ion cloud morphology in the CRRES plasma injection experiments, J. Geophys. Res., 106, 21,289.

Denton, M. H., M. F. Thomsen, H. Korth, S. Lynch, J.-C. Zhang, and M. W. Liemohn (2005), Bulk plasma properties at geosynchronous orbit, J. Geophys. Res., 110, A07223, doi:10.1029/2004JA010861.

Denton, M. H., J. E. Borovsky, R. M. Skoug, M. F. Thomsen, B. Lavraud, M. G. Henderson, R. L. McPherron, J.-C. Zhang, and M. W. Liemohn (2006), Geomagnetic disturbances driven by ICME- and CIR- dominated solar wind from 1994 to 2002, J. Geophys. Res., 111, A07S07, doi:10.1029/2005JA011436.

De Zeeuw, D. L., T. I. Gombosi, C. P. T. Groth, K. G. Powell, and Q. F. Stout (2000), An adaptive MHD method for global space weather simulations, IEEE Trans. Plasma Sci., 28, 1956.

De Zeeuw, D. L., S. Sazykin, R. A. Wolf, T. I. Gombosi, A. J. Ridley, and G. Tóth (2004), Coupling of a global MHD code and an inner magnetospheric model: Initial results, J. Geophys. Res., 109, A12219, doi:10.1029/2003JA010366.

Dungey, J. W. (1961), Interplanetary magnetic field and the auroral zone, Phys. Rev. Lett., 6, 47.

Eastman, T. E., E. W. Hones Jr., S. J. Bame, and J. R. Asbridge (1976), The magnetospheric boundary layer: Site of plasma, momentum, and energy transfer from the magnetosheath into the magnetosphere, Geophys. Res. Lett., 3, 685 .

Ebihara, Y., and M. Ejiri (2000), Simulation study on fundamental properties of the storm-time ring current, J. Geophys. Res., 105, 15,843.

Ebihara, Y., and M.-C. Fok (2004), Postmidnight storm-time enhancement of tens-of-keV proton flux, J. Geophys. Res., 109, A12209, doi:10.1029/ 2004JA010523.

Ebihara, Y., M.-C. Fok, R. A. Wolf, M. F. Thomsen, and T. E. Moore (2005), Nonlinear impact of plasma sheet density on the storm-time ring current, J. Geophys. Res., 110, A02208, doi:10.1029/2004JA010435.

Fok, M.-C., and T. E. Moore (1997), Ring current modeling in a realistic magnetic field configuration, Geophys. Res. Lett., 24, 1775.

Fok, M.-C., J. U. Kozyra, and A. F. Nagy (1991), Lifetime of ring current particles due to Coulomb collisions in the plasmasphere, J. Geophys. Res., 96, 7861.

Frank, L. A. (1967), On the extraterrestrial ring current during geomagnetic storms, J. Geophys. Res, 72, 3753.

Frank, L. A., K. L. Ackerson, W. R. Paterson, J. A. Lee, M. R. English, and G. L. Pickett (1994), The Comprehensive Plasma Instrumentation (CPI) for the Geotail spacecraft, J. Geomagn. Geoelectr., 46, 23.

Fujimoto, M., A. Nishida, T. Mukai, Y. Saito, T. Yamamoto, and S. Kokubun (1996), Plasma entry from the flanks of the near-Earth magnetotail: GEOTAIL observations in the dawnside LLBL and the plasma sheet, J. Geomagn. Geoelectr., 48, 711 .

Fujimoto, M., T. Mukai, H. Kawano, M. Nakamura, A. Nishida, Y. Saito, T. Yamamoto, and S. Kokubun (1998a), Structure of the low-latitude boundary layer: A case study with Geotail data, J. Geophys. Res., 103, 2297. 
Fujimoto, M., T. Terasawa, T. Mukai, Y. Saito, T. Yamamoto, and S. Kokubun (1998b), Plasma entry from the flanks of the near-Earth magnetotail: Geotail observations, J. Geophys. Res., 103, 4391.

Fuselier, S. A., et al. (2003), Cusp dynamics and ionospheric outflow, Space Sci. Rev., 109, 285.

Gonzalez, W. D., and B. T. Tsurutani (1987), Criteria of interplanetary parameters causing intense magnetic storms (Dst $<-100 \mathrm{nT})$, Planet. Space Sci., 35, 1101.

Gonzalez, W. D., J. A. Joselyn, Y. Kamide, H. W. Kroehl, G. Rostoker, B. T. Tsurutani, and V. M. Vasyliunas (1994), What is a geomagnetic storm?, J. Geophys. Res., 99, 5771.

Hamilton, D. C., G. Gloeckler, F. M. Ipavich, W. Stüdemann, B. Wilken, and G. Kremser (1988), Ring current development during the great geomagnetic storm of February 1986, J. Geophys. Res, 93, 14,343.

Heppner, J. P. (1972), Polar cap electric field distributions related to the interplanetary magnetic field direction, J. Geophys. Res., 77, 4877.

Iijima, T., and T. A. Potemra (1976), The amplitude distribution of fieldaligned currents at northern high latitudes observed by TRIAD, J. Geophys. Res., 81, 2165.

Iyemori, T., and D. R. K. Rao (1996), Decay of the Dst component of geomagnetic disturbance after substorm onset and its implication to storm substorm relation, Ann. Geophys., 14, 608

Janhunen, P. (1996), GUMICS-3: A global ionosphere-magnetosphere coupling simulation with high ionospheric resolution, in Proceedings of the ESA 1996 Symposium on Environment Modelling for Space-Based Applications, ESA SP-392, pp. 233-239, Eur. Space Agency, Paris.

Jordanova, V. K., L. M. Kistler, J. U. Kozyra, G. V. Khazanov, and A. F. Nagy (1996), Collisional losses of ring current ions, J. Geophys. Res. $101,111$.

Jordanova, V. K., A. Boonsiriseth, R. M. Thorne, and Y. Dotan (2003), Ring current asymmetry from global simulations using a high-resolution electric field model, J. Geophys. Res., 108(A12), 1443, doi:10.1029/ 2003JA009993.

Kistler, L. M., F. M. Ipavich, D. C. Hamilton, G. Gloeckler, B. Wilken, G. Kremser, and W. Stüdemann (1989), Energy spectra of the major ion species in the ring current during geomagnetic storms, J. Geophys. Res., 94, 3579 .

Kokubun, S., T. Yamamoto, M. H. Acuna, K. Hayashi, K. Shiokawa, and H. Kawano (1994), The Geotail Magnetic Field Experiment, J. Geomagn. Geoelectr., 46, 7.

Korth, H., and M. F. Thomsen (2001), Plasma sheet access to geosynchronous orbit: Generalization to numerical global field models, J. Geophys. Res., 106, 29,655

Korth, H., M. F. Thomsen, J. E. Borovsky, and D. J. McComas (1999), Plasma sheet access to geosynchronous orbit, J. Geophys. Res., 104 25,047 .

Kozyra, J. U., M.-C. Fok, E. R. Sanchez, D. S. Evans, D. C. Hamilton, and A. F. Nagy (1998a), The role of precipitation losses in producing the rapid early recovery phase of the Great Magnetic Storm of February 1986, J. Geophys. Res., 103, 6801.

Kozyra, J. U., V. K. Jordanova, J. E. Borovsky, M. F. Thomsen, D. J. Knipp, D. S. Evans, D. J. McComas, and T. E. Cayton (1998b), Effects of a high-density plasma sheet on ring current development during the November 2-6, 1993, magnetic storm, J. Geophys. Res., 103, 26,285.

Kozyra, J. U., M. W. Liemohn, C. R. Clauer, A. J. Ridley, M. F. Thomsen, J. E. Borovsky, J. L. Roeder, V. K. Jordanova, and W. D. Gonzalez (2002), Multistep Dst development and ring current composition changes during the 4-6 June 1991 magnetic storm, J. Geophys. Res., 107(A8), 1224, doi:10.1029/2001JA000023.

Kremser, G., W. Stüdemann, B. Wilken, G. Gloeckler, D. C. Hamilton, and F. M. Ipavich (1987), Average spatial distribution of energetic $\mathrm{O}^{+}, \mathrm{O}^{2+}$ $\mathrm{O}^{6+}$, and $\mathrm{C}^{6+}$ ions in the magnetosphere observed by AMPTE/CCE, J. Geophys. Res., 92, 4459.

Lennartsson, O. W. (1997), ISEE ion composition data with implications for solar wind entry into Earth's magnetotail, Space Sci. Rev, 80, 305.

Liemohn, M. W., A. J. Ridley, P. C. Brandt, D. L. Gallagher, J. U. Kozyra, D. M. Ober, D. G. Mitchell, E. C. Roelof, and R. DeMajistre (2005), Parametric analysis of nightside conductance effects on inner magnetospheric dynamics for the 17 April 2002 storm, J. Geophys. Res., 110, A12S22, doi:10.1029/2005JA011109.

Lu, G., et al. (1994), Interhemispheric asymmetry of the high-latitude ionospheric convection pattern, J. Geophys. Res., 99, 6491.

Lui, A. T. Y., R. W. McEntire, and S. M. Krimigis (1987), Evolution of the ring current during two geomagnetic storms, J. Geophys. Res., 92, 7459.

Lukianova, R., and F. Christiansen (2006), Modeling of the global distribution of ionospheric electric fields based on realistic maps of field-aligned currents, J. Geophys. Res., 111, A03213, doi:10.1029/2005JA011465.

Lyon, J. G., J. Fedder, and J. Huba (1986), The effect of different resistivity models on magnetotail dynamics, J. Geophys. Res., 91, 8057.
McComas, D. J., S. J. Bame, B. L. Barraclough, J. R. Donart, R. C. Elphic, J. T. Gosling, M. B. Moldwin, K. R. Moore, and M. F. Thomsen (1993), Magnetospheric plasma analyzer: Initial 3-spacecraft observations from geosynchronous orbit, J. Geophys. Res., 98, 13,453.

Nishikawa, K.-I. (1997), Particle entry into the magnetosphere with a southward interplanetary magnetic field studied by a three-dimensional electromagnetic particle code, J. Geophys. Res., 102, 17,631.

Nishikawa, K. (2001), Global particle simulation study of substorm onset and particle acceleration, Space Sci. Rev., 95(1-2), 361.

Nopper, R. W., Jr., and R. L. Carovillano (1978), Polar-equatorial coupling during magnetically active periods, Geophys. Res. Lett., 5, 699

Ober, D. M., N. C. Maynard, and W. J. Burke (2003), Testing the Hill model of transpolar potential saturation, J. Geophys. Res., 108(A12), 1467, doi:10.1029/2003JA010154.

Ogino, T., and R. J. Walker (1984), A magnetohydrodynamic simulation of the bifurcation of tail lobes during intervals with a northward interplanetary magnetic field, Geophys. Res. Lett., 11, 1018

Papitashvili, V. O., and F. J. Rich (2002), High-latitude ionospheric convection models derived from Defense Meteorological satellite Program ion drift observations and parameterized by the interplanetary magnetic field strength and direction, J. Geophys. Res., 107(A8), 1198 , doi:10.1029/2001JA000264.

Papitashvili, V. O., F. Christiansen, and T. Neubert (2002), A new model of field-aligned currents derived from high-precision satellite magnetic field data, Geophys. Res. Lett., 29(14), 1683, doi:10.1029/2001GL014207.

Powell, K. G., P. L. Roe, T. J. Linde, T. I. Gombosi, and D. L. De Zeeuw (1999), A solution-adaptive upwind scheme for ideal magnetohydrodynamics, J. Comput. Phys., 154(2), 284

Raeder, J., R. J. Walker, and M. Ashour-Abdalla (1995), The structure of the distant geomagnetic tail during long periods of northward IMF, Geophys. Res. Lett., 22, 349

Richmond, A. D., and Y. Kamide (1988), Mapping electrodynamic features of the high-latitude ionosphere from localized observations: Technique, J. Geophys. Res., 93, 5741

Ridley, A. J., D. L. De Zeeuw, T. I. Gombosi, and K. G. Powell (2001) Using steady-state MHD results to predict the global state of the magnetosphere-ionosphere system, J. Geophys. Res., 106, 30,06730,076

Ridley, A. J., T. I. Gombosi, and D. I. De Zeeuw (2004), Ionosphere control of the magnetosphere: Conductance, Ann. Geophys., 22, 567-584.

Rothwell, P. L., and J. R. Jasperse (2006), Modeling the connection of the global ionospheric electric fields to the solar wind, J. Geophys. Res., 111, A03211, doi:10.1029/2004JA010992.

Sazykin, S. Y. (2000), Theoretical studies of penetration of magnetospheric electric fields to the ionosphere, Ph.D. thesis, Utah State Univ., Logan, Utah.

Shelley, E. G., R. G. Johnson, and R. D. Sharp (1972), Satellite observations of energetic heavy ions during a geomagnetic storm, J. Geophys Res., 77, 6104.

Shue, J.-H., et al. (1998), Magnetopause location under extreme solar wind conditions, J. Geophys. Res., 103, 17,691-17,700

Siscoe, G. L., G. M. Erickson, B. U. O. Sonnerup, N. C. Maynard, J. A Schoendorf, K. D. Siebert, D. R. Weimer, W. W. White, and G. R. Wilson (2002), Hill model of transpolar potential saturation: Comparisons with MHD simulations, J. Geophys. Res., 107(A6), 1075, doi:10.1029/ 2001JA000109.

Smith, P. H., N. K. Bewtra, and R. A. Hoffman (1979), Motions of charged particles in the magnetosphere under the influence of a time-varying large scale convection electric field, in Quantitative Modeling of Magnetospheric Processes, Geophys. Monogr. Ser., vol. 21, edited by W. P. Olsen, pp. 513-534, AGU, Washington, D. C.

Song, P., and C. T. Russell (1992), Model of the formation of the lowlatitude boundary layer for strongly northward interplanetary magnetic field, J. Geophys. Res., 97, 1411-1420.

Sugiura, M., and T. Kamei (1991), Equatorial DST index 1957-1986 in IAGA Bull. 40, edited by A. Berthelier and M. Menvielle, Int. Serv. of Geomagn. Indices Publ. Off., Saint Maur, France.

Taguchi, S., M. Sugiura, J. D. Winningham, and J. A. Slavin (1993), Characterization of the IMF $B_{Y}$-dependent field-aligned currents in the cleft region based on DE2 observations, J. Geophys. Res., 98, 1393.

Tanaka, T. (1995), Generation mechanisms for magnetosphere-ionosphere current systems deduced from a three-dimensional MHD simulation of the solar wind-magnetosphere-ionosphere coupling process, J. Geophys. Res., 100, 12,057-12,074

Thomsen, M. F., J. E. Borovsky, D. J. McComas, and M. B. Moldwin (1996), Observations of the Earth's plasma sheet at geosynchronous orbit, in Proceedings of the 10th Taos Workshop on the Earth's Trapped Particle Environment, edited by G. D. Reeves, AIP Conf. Proc. 383, 25.

Thomsen, M. F., J. E. Borovsky, D. J. McComas, R. C. Elphic, and S. Maurice (1998a), The magnetospheric response to the CME passage 
of January $10-11,1997$, as seen at geosynchronous orbit, Geophys. Res. Lett., 25, 2545-2548.

Thomsen, M. F., J. E. Borovsky, D. J. McComas, and M. R. Collier (1998b), Variability of the ring current source population, Geophys. Res. Lett., 25, 3481-3484.

Thomsen, M. F., J. E. Borovsky, R. M. Skoug, and C. W. Smith (2003), Delivery of cold, dense plasma sheet material into the near-Earth region, J. Geophys. Res., 108(A4), 1151, doi:10.1029/2002JA009544.

Toffoletto, F., S. Sazykin, R. Spiro, and R. Wolf (2003), Inner magnetospheric modeling with the Rice Convection Model, Space Sci. Rev., 107, $175-196$

Tóth, G., et al. (2004), A physics-based software framework for Sun-Earth connection modeling, in Multiscale Coupling of Sun-Earth Processes, edited by A. T. Y. Lui, Y. Kamide, and G. Consolini, pp. 383-397, Elsevier, New York.

Tóth, G., et al. (2005), Space Weather Modeling Framework: A new tool for the space science community, J. Geophys. Res., 110, A12226, doi:10.1029/2005JA011126.

Wanliss, J. A., and K. M. Showalter (2006), High-resolution global storm index: Dst versus SYM-H, J. Geophys. Res., 111, A02202, doi:10.1029/ 2005JA011034

Waters, C. L., B. J. Anderson, and K. Liou (2001), Estimation of global field aligned currents using the Iridium ${ }^{\mathbb{R}}$ system magnetometer data, Geophys. Res. Lett., 28, 2165-2168.

Weimer, D. R. (1995), Models of high-latitude electric potentials derived with a least error fit of spherical harmonic coefficients, J. Geophys. Res., $100,19,595-19,607$

Weimer, D. R. (2001), An improved model of ionospheric electric potentials including substorm perturbations and applications to the Geospace Environment Modeling November 24, 1996 event, J. Geophys. Res., 106, 407-416.

Weimer, D. R. (2004), Correction to "Predicting interplanetary magnetic field (IMF) propagation delay times using the minimum variance technique," J. Geophys. Res., 109, A12104, doi:10.1029/2004JA010691.
Weimer, D. R., D. M. Ober, N. C. Maynard, M. R. Collier, D. J. McComas, N. F. Ness, C. W. Smith, and J. Watermann (2003), Predicting interplanetary magnetic field (IMF) propagation delay times using the minimum variance technique, J. Geophys. Res., 108(A1), 1026, doi:10.1029/ 2002JA009405.

White, W. W., G. L. Siscoe, G. M. Erickson, Z. Kaymaz, N. C. Maynard, K. D. Siebert, B. U. Ö. Sonnerup, and D. R. Weimer (1998), The magnetospheric sash and the cross-tail S, Geophys. Res. Lett., 25, 1605-1608.

Winglee, R. M. (1994), Non-MHD influences on the magnetospheric current system, J. Geophys. Res., 99, 13,437-13,454.

Winglee, R. M. (1998), Multi-fluid simulations of the magnetosphere: The identification of the geopause and its variation with IMF, Geophys. Res. Lett., 25, 4441-4444.

Wolf, R. A., R. W. Spiro, and F. J. Rich (1991), Extension of the Rice Convection Model into the high-latitude ionosphere, J. Atmos. Terr. Phys., 53, 817-829.

Zhang, J.-C., M. W. Liemohn, J. U. Kozyra, B. J. Lynch, and T. H. Zurbuchen (2004), A statistical study of the geoeffectiveness of magnetic clouds during high solar activity years, J. Geophys. Res., 109, A09101, doi:10.1029/2004JA010410.

Zhang, J.-C., M. W. Liemohn, M. F. Thomsen, J. U. Kozyra, M. H. Denton, and J. E. Borovsky (2006), A statistical comparison of hot-ion properties at geosynchronous orbit during intense and moderate geomagnetic storms at solar maximum and minimum, J. Geophys. Res., 111, A07206, doi:10.1029/2005JA011559.

J. E. Borovsky and M. F. Thomsen, Los Alamos National Laboratory, MS D466, Los Alamos, NM 87545, USA.

D. L. De Zeeuw, T. I. Gombosi, J. U. Kozyra, M. W. Liemohn, A. J. Ridley, and G. Toth, Center for Space Environment Modeling, University of Michigan, 2455 Hayward Street, Ann Arbor, MI 48109-2143, USA.

S. Sazykin, R. A. Wolf, and J. Zhang, Department of Physics and Astronomy, Rice University, MS-108, 6100 S. Main Street, Houston, TX 77005-1892, USA. (jichunz@rice.edu) 